\title{
Mitochondria as a therapeutic target for cardiac ischemia-reperfusion injury (Review)
}

\author{
WENWEN MARIN ${ }^{1}$, DENNIS MARIN ${ }^{2}$, XIANG AO $^{3}$ and YING LIU ${ }^{1,3}$ \\ ${ }^{1}$ Institute for Translational Medicine, The Affiliated Hospital of Qingdao University, College of Medicine, Qingdao University, \\ Qingdao, Shandong 266071; ${ }^{2}$ Qingdao University of Science and Technology, Qingdao, Shandong 266061; \\ ${ }^{3}$ School of Basic Medical Sciences, College of Medicine, Qingdao University, Qingdao, Shandong 266071, P.R. China
}

Received June 8, 2020; Accepted November 18, 2020

DOI: $10.3892 /$ ijmm.2020.4823

\begin{abstract}
Acute myocardial infarction is the leading cause of cardiovascular-related mortality and chronic heart failure worldwide. As regards treatment, the reperfusion of ischemic tissue generates irreversible damage to the myocardium, which is termed 'cardiac ischemia-reperfusion (IR) injury'. Due to the large number of mitochondria in cardiomyocytes, an increasing number of studies have focused on the roles of mitochondria in IR injury. The primary causes of IR injury are reduced oxidative phosphorylation during hypoxia and the increased production of reactive oxygen species (ROS), together with the insufficient elimination of these oxidative species following reperfusion. IR injury includes the oxidation of DNA, incorrect modifications of proteins, the disruption of the mitochondrial membrane and respiratory chain, the loss of mitochondrial membrane potential $(\Delta \Psi \mathrm{m}), \mathrm{Ca}^{2+}$ overload, mitochondrial permeability transition pore formation, swelling of the mitochondria, and ultimately, cardiomyocyte necrosis. The present review article discusses the molecular mechanisms of IR injury, and summarizes the metabolic and dynamic changes occurring in the mitochondria in response to IR stress. The mitochondria are strongly recommended as a target for the development of therapeutic agents; however, the appropriate use of agents remains a challenge.
\end{abstract}

\section{Contents}

1. Introduction

2. Molecular mechanisms underlying IR injury

Correspondence to: Dr Wenwen Marin or Dr Ying Liu, Institute for Translational Medicine, The Affiliated Hospital of Qingdao University, College of Medicine, Qingdao University, 308 Ningxia Road, Qingdao, Shandong 266071, P.R. China

E-mail: wenwen.marin@gmx.de

E-mail: liuying_hero@163.com

Key words: mitochondria, ischemia-reperfusion injury, oxidative stress, myocardial infarction, therapeutic agent
3. Alterations in mitochondrial metabolism during cardiac IR injury

4. Mitochondrial dynamics in response to IR stress

5. Current developments in mitochondria-targeted agents with cardioprotective effects against IR injury

6. Future perspectives

7. Conclusions

\section{Introduction}

Ischemia is the disruption of blood flow to tissues, which also results in the reduction of delivery of oxygen and metabolites to cells (1). Pathological ischemia in the heart results in oxidative damage, apoptosis and cardiomyocyte death, which is termed myocardial infarction (MI) (2). The standard approach for the management of a patient with MI is to restore blood flow to the ischemic tissue as quickly as possible. However, this restoration of oxygenated blood causes extensive tissue damage, termed ischemia-reperfusion (IR) injury or hypoxia-reoxygenation injury $(3,4)$, which may result in morbidity and mortality in the cardiac intervention following a heart attack or stroke $(5,6)$, as well as in other pathological situations, such as acute kidney injury, muscle injury, organ transplantation, hypovolemic shock and elective surgery $(5,7)$. MI followed by reperfusion is the major stressor leading to chronic heart failure (8). However, reducing the irreversible ischemic damage due to MI and the inevitable IR injury that occurs during the reperfusion of ischemic tissues remains a challenge for cardiac therapy.

The mitochondria occupy $>30 \%$ of the cardiomyocyte volume, provide $95 \%$ of adenosine triphosphate (ATP) generated through oxidative phosphorylation (OXPHOS) for the beating of the heart, and serve as the metabolic hub for the citric acid cycle and fatty acid $\beta$-oxidation $(3,8,9)$. IR damages the mitochondrial respiratory chain, and accompanied by ATP depletion, results in the accumulation of mitochondrial reactive oxygen species (ROS; mtROS; ROS produced by mitochondria), and oxidative damage on mitochondrial DNA (mtDNA) or proteins, ultimately leading to necrotic and apoptotic myocardial cell death. The extent of mitochondrial damage is a key determinant in the progression of myocardial IR injury towards to heart failure (10). 


\section{Molecular mechanisms underlying IR injury}

In the normoxic heart (Fig. 1A), glucose and fatty acids are oxidized through glycolysis, $\beta$-oxidation and the tricarboxylic acid (TCA) cycle, respectively. The electrons from the final metabolites nicotinamide adenine dinucleotide reduced form $\left(\mathrm{NADH} ; \mathrm{NAD}^{+}+\mathrm{H}^{+}+2 \mathrm{e}^{-} \leftrightarrow \mathrm{NADH}\right)$ and flavin adenine dinucleotide hydroquinone form $\left(\mathrm{FADH}_{2} ; \mathrm{FAD}+2 \mathrm{H}^{+}+2 \mathrm{e}^{-} \leftrightarrow\right.$ $\left.\mathrm{FADH}_{2}\right)$ reduce oxygen $\left(\mathrm{O}_{2}\right)$ into water through the electron transport chain in the mitochondrial cristae membrane and generate ATP through OXPHOS driven by the protonmotive force $(\Delta \mathrm{p})(11)$. During the conventional forward electron transport, $2 \mathrm{e}^{-}$are transferred from NADH to reduce coenzyme Q (CoQ, ubiquinone) into $\mathrm{CoQH}_{2}$ (the fully reduced form of $\mathrm{CoQ}$, ubiquinol), following pumping of 4 protons $\left(4 \mathrm{H}^{+}\right)$ into the mitochondrial intermembrane space to maintain the $\Delta \mathrm{p}$ for the later ATP synthesis. If complex I is inhibited by the $\mathrm{CoQ}$ inhibitor rotenone, the flavin mononucleotide (FMN) can accept the electrons from NADH and reduce $\mathrm{O}_{2}$ to generate superoxide $\left(\mathrm{O}_{2}^{-}\right)$at complex I $(5,12)$.

Under hypoxic conditions (Fig. 1B), the levels of the metabolites lactate and succinate are significantly increased in ischemic tissues. The accumulation of succinate is a primary metabolic signature of ischemia $(13,14)$, and is considered to be a consequence of the inhibition of the TCA cycle during ischemia or anoxia. However, recent studies have proposed that it may also be due to the reversal of succinate dehydrogenase (SDH), driven by the electrons transported from the accumulated $\mathrm{CoQH}_{2}$ to fumarate $(14,15)$. Moreover, succinate cannot be further converted into succinyl-coenzyme A (succinyl-CoA) by succinyl-CoA synthetase due to the depletion of GTP and CoA during ischemia $(13,14)$.

During the reperfusion process (Fig. 1C), the accumulated succinate is rapidly re-oxidized by the SDH in the mitochondrial respiratory chain complex II, resulting in a burst of superoxide production from complex I by reverse electron transport (RET). A $\mathrm{CoQH}_{2}$ pool and a high $\Delta \mathrm{p}$ are required to drive RET. The accumulation of $\mathrm{CoQH}_{2}$ is formed during hypoxia and the high $\Delta \mathrm{p}$ is restored from proton pumping by complexes III and IV within seconds of reoxygenation. RET occurs when electrons are forced to flow backward from a reduced CoQ pool through complex I and onto the FMN to drive superoxide formation. The superoxide generated at complex I by RET upon reperfusion is considered as the major source of ROS during IR injury $(5,14,15)$. In addition to the classic theory described above, there are also other sources of mtROS which contribute to IR injury, which have been elucidated in recent years, such as the ROS formed by monoamine oxidases, p66 $^{\text {Shc }}$ protein and NADH oxidases $(16,17)$.

ROS include free superoxide anions $\left(\mathrm{O}_{2}{ }^{-*}\right)$, hydroxyl radicals $(\mathrm{OH} \cdot)$, non-radical hydrogen peroxide $\left(\mathrm{H}_{2} \mathrm{O}_{2}\right)$ and peroxynitrite $\left(\mathrm{ONOO}^{-}\right)$(18). Usually, the superoxide anion generated in the mitochondrial matrix can be converted by the manganese-dependent superoxide dismutase (MnSOD, also known as SOD2) into $\mathrm{H}_{2} \mathrm{O}_{2}$, and then further detoxified by glutathione peroxidase and thioredoxin enzymes into $\mathrm{H}_{2} \mathrm{O}$. The superoxide anion $\left(\mathrm{O}_{2}^{\left.-{ }^{-}\right)}\right.$diffuses into the cytoplasm and is transformed by copper-zinc-dependent SOD (CuZnSOD, also known as SOD1) into $\mathrm{H}_{2} \mathrm{O}_{2}(19,20)$. When the production of ROS exceeds the defensive capacity of cellular antioxidant systems, the excessive ROS cause functional and structural damage to the cells (21).

Cardiomyocyte oxidative damage is the major consequence of cardiac IR injury; however, some damage can be attributed to circulating platelets. During cardiac ischemia, activated platelets induce thrombi formation and occlusion of microcirculation, which causes secondary ischemia injury after reperfusion $(4,22)$.

\section{Alterations in mitochondrial metabolism during cardiac IR injury}

Oxidative stress. Restoring blood flow and the reoxygenation of the cardiac tissue following myocardial ischemia generates excessive quantities of ROS. The accumulation of ROS damages cellular components (such as proteins, lipid and DNA oxidation), increases the susceptibility of mitochondria and cells, and accelerates aging and cell death $(3,12,21,23)$. For example, toxic ROS can damage mitochondrial respiratory complex I via the oxidation of thiols, which in turn results in the production of excess ROS during reperfusion (6). The phospholipid cardiolipin, which is required for the mitochondrial inner membrane, and the activities of complexes III and IV in the respiratory chain, contains a high percentage of unsaturated fatty acids and is sensitive to peroxidation by ROS (24). Cardiolipin is also essential in the biochemical functions of several mitochondrial proteins, such as mitochondrial creatine kinase (mitCK) (24). mitCK plays an important role in the creatine-phosphocreatine energy transferring system (25). The mitCK system has been shown to be damaged by cardiac ischemia and oxidative stress (26). The burst of ROS from mitochondria during IR not only causes direct acute damage in cells or tissues, but also initiates the long-term inflammatory response following reperfusion or persistent pathologies such as in chronic heart diseases $(7,27)$.

Carbon stress. During IR, activated acyl-CoA accumulates in the mitochondrial matrix due to a defective $\beta$-oxidation pathway. The non-enzymatic acylation of lysine residues by acyl-CoA causes matrix protein dysfunction and aggregation (28-30).

Depletion of $N A D^{+}$. A damaged mitochondrial respiratory chain also causes the depletion of $\mathrm{NAD}^{+}$. Since NAD is required for ATP synthesis and also for the deacylation of Lys by sirtuins, $\mathrm{NAD}^{+}$depletion disrupts the pathways for bioenergetics and also the pathways altered by carbon stress $(30,31)$.

Bioenergetic insufficiency (ATP). Mitochondria generate ATP through OXPHOS within the electron transport chain at the mitochondrial inner membrane. The lack of oxygen and respiratory substrates impairs OXPHOS during ischemia (3). IR injury markedly increases mitochondrial permeability, and dissipates electron and proton gradients. Furthermore, mitochondrial electron transport chain subunits lose their integrity and are degraded under conditions of IR. OXPHOS is markedly diminished in the abnormal mitochondria, leading to bioenergetic insufficiency during MI (23).

Formation of mitochondrial permeability transition pore $(m P T P)$. $\mathrm{mPTP}$ is a large conductance channel in the inner membrane, which is activated by $\mathrm{Ca}^{2+}$ accumulation $(32,33)$. 


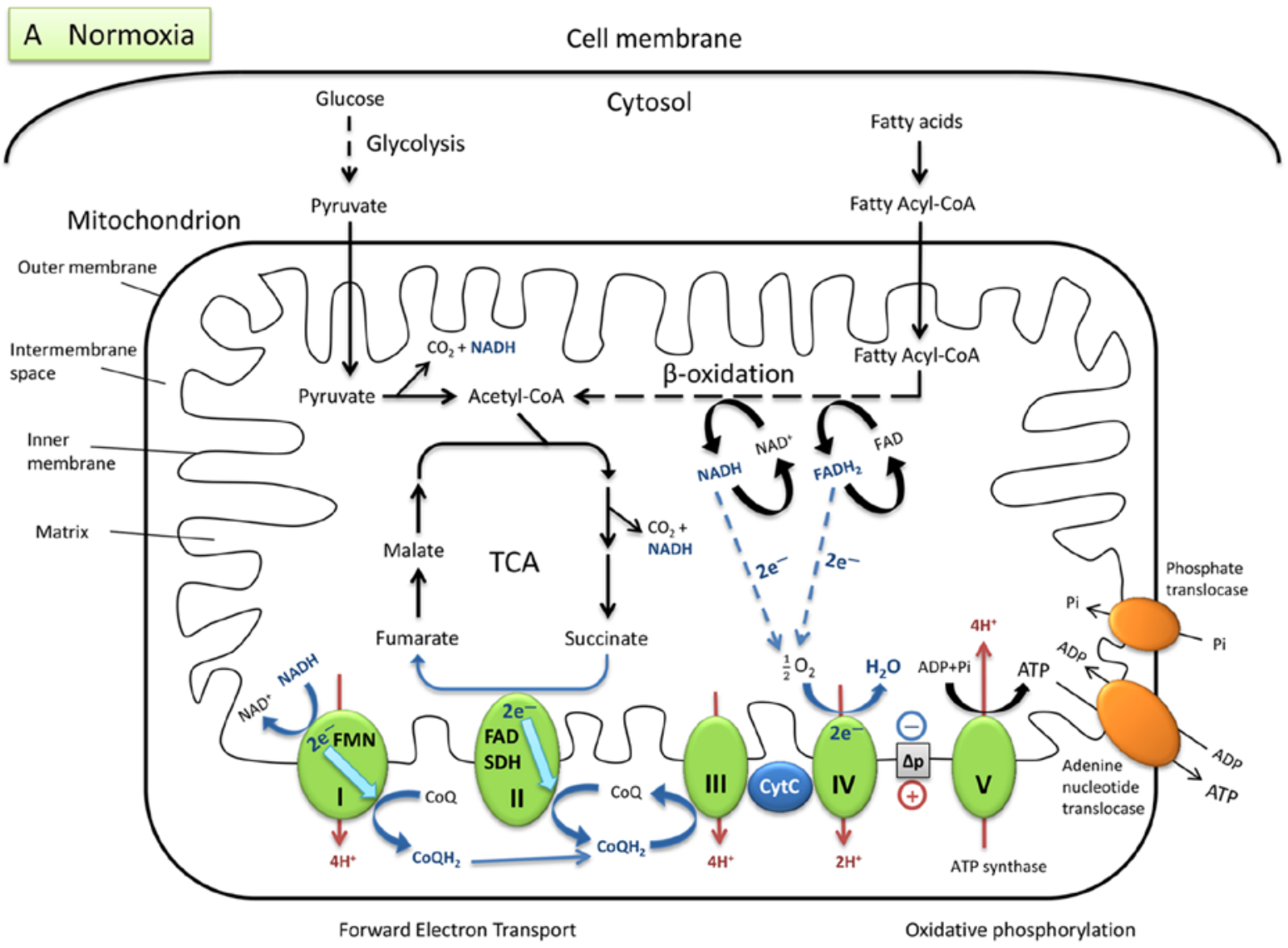

\section{B Ischemia} Cell membrane

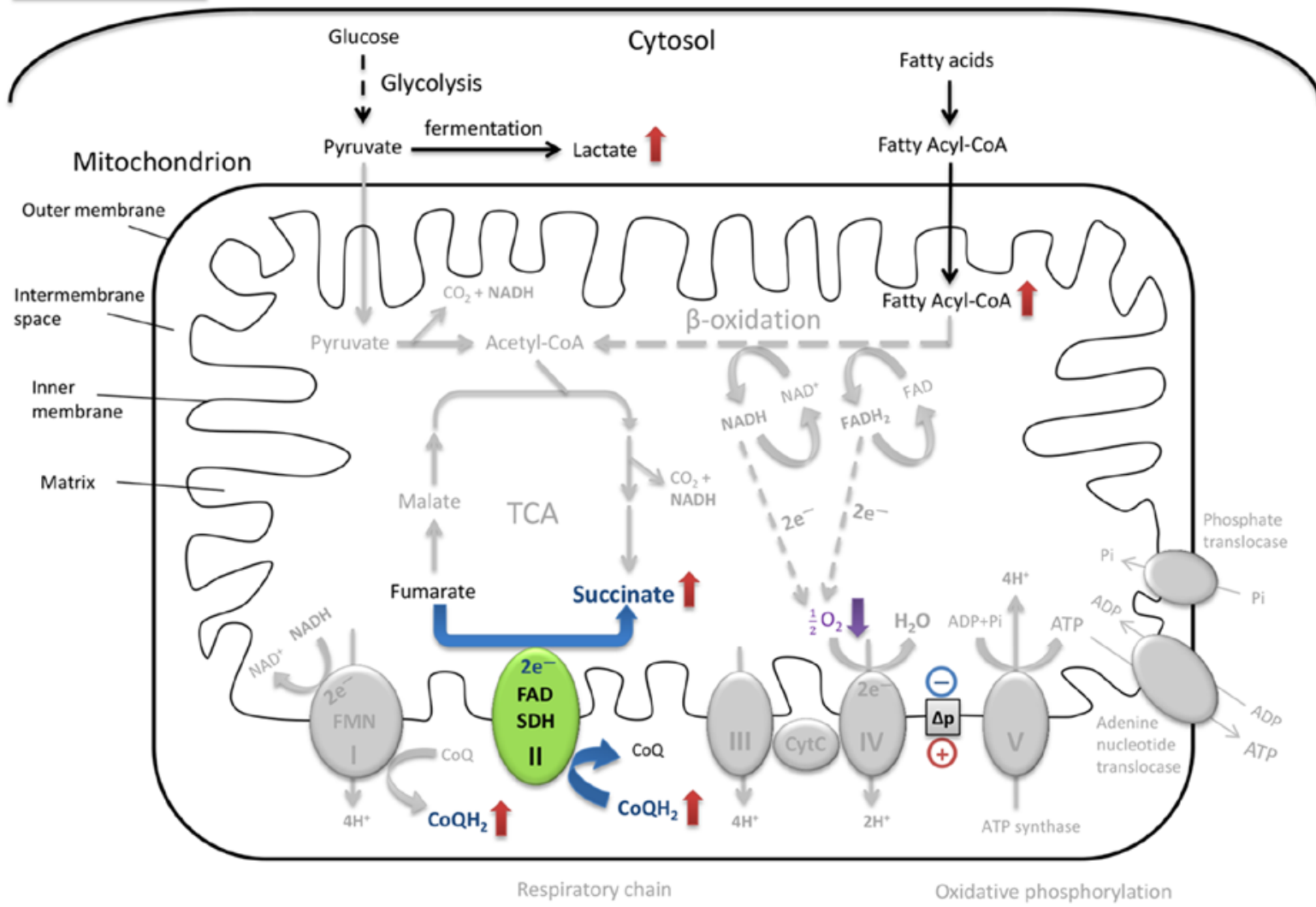

Figure 1. Continued. 


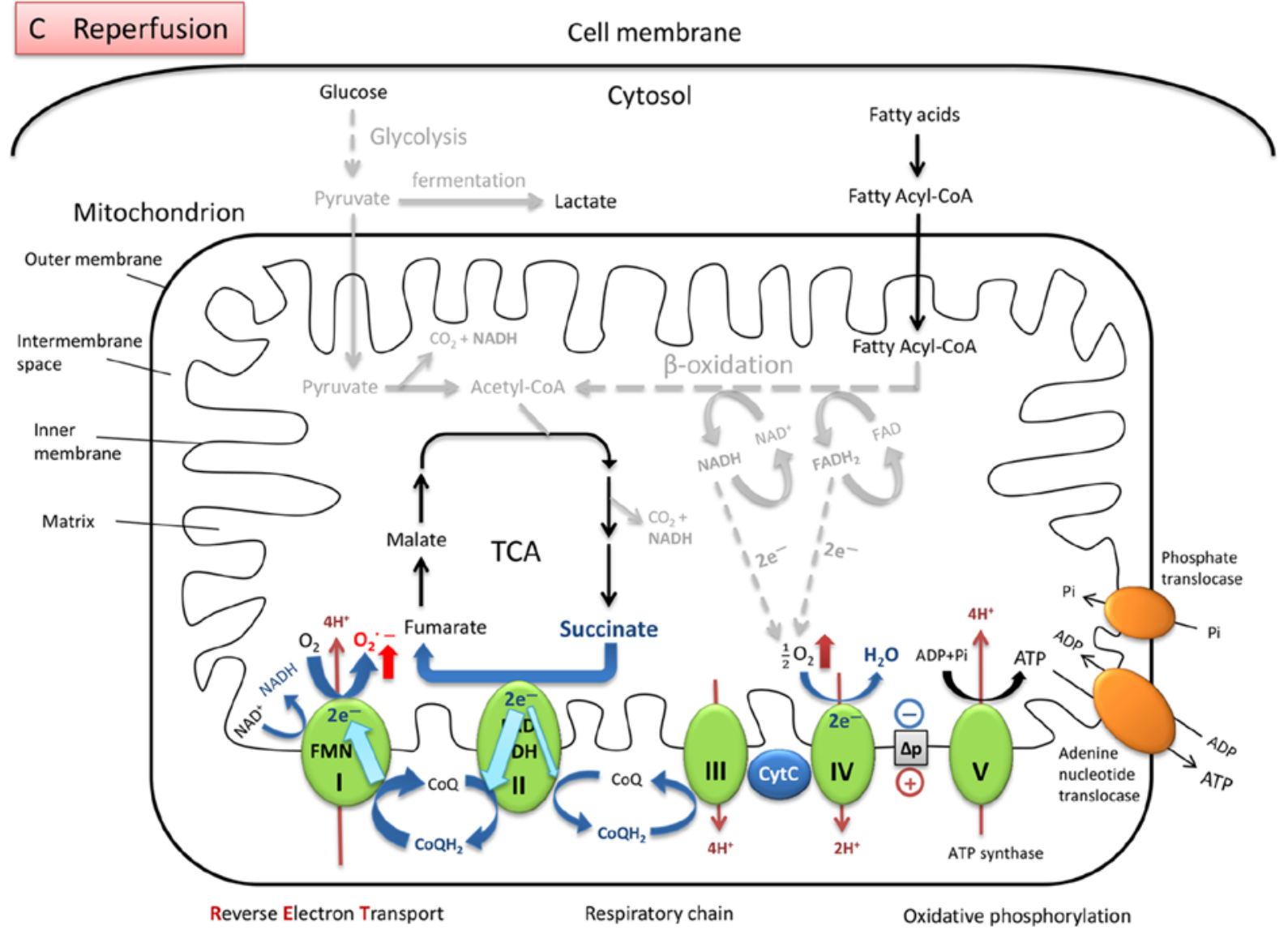

Figure 1. Brief illustration of metabolism via aerobic respiration under normal conditions and the mechanism of mtROS production during ischemia-reperfusion in heart cells. (A) Under normal oxygen levels, metabolism is balanced by a series of hydrogen and electron transfer reactions in the respiratory chain (B) During ischemia, the oxygen levels are dramatically decreased, and electron transport flow is diminished. Ubiquinone (CoQ) accepts an electron and transforms into ubiquinol $\left(\mathrm{CoQH}_{2}\right)$ at complex I. SDH catalyzes the reverse reaction by utilizing the increased levels of $\mathrm{CoQH}_{2}$ to reduce fumarate to succinate. This results in the accumulation of succinate which serves as the final electron sink instead of oxygen. (C) When reperfusion occurs, and oxygen supply is restored, the succinate is rapidly oxidized into fumarate by forward catalysis by SDH. Due to the slow reestablishment of ATP synthesis, the electrons from the excess flux of $\mathrm{CoQH}_{2}$ are forced backward through FMN in complex I. Production of superoxide is significantly increased, generated from oxygen reduction, driven by RET together with the high proton-motive force. mtROS, mitochondrial reactive oxygen species; TCA, tricarboxylic acid cycle; I, complex I; FMN, flavin mononucleotide; II, complex II; SDH, succinate dehydrogenase; FAD, flavin adenine dinucleotide; III, complex III; CytC, cytochrome $c$; IV, complex IV; $\mathrm{V}$, complex V (also termed ATP synthase).

Ischemic oxidative damage results in the marked reduction of mitochondrial membrane potential $(\Delta \Psi \mathrm{m})$, mitochondrial $\mathrm{Ca}^{2+}$ bursts and the opening of the non-selective mPTP in the inner membrane. Accompanied by the release of matrix proteins and mtDNA into the cytosol, mPTP opening increases colloid-osmotic pressure in the matrix, induces the innate immune response, and initiates the activation of proteases and lipases, leading to mitochondrial swelling and cardiomyocyte death through necrosis $(34,35)$. The excessive activation of the mitochondrial fission protein, dynamin-related protein 1 (Drp1), also facilitates mPTP opening and accelerates cell death. The genetic or pharmacological inhibition of Drp1 has been shown to decrease the frequency of transient opening of mPTP during cardiac IR and reduce the infarct size following MI $(8,36,37)$.

Calcium overload. During ischemia, ATP depletion and acidosis caused by the increasing lactate levels drive cytosolic $\mathrm{Na}^{+}$accumulation through the plasma membrane $\mathrm{Na}^{+} / \mathrm{H}^{+}$ exchanger, which, in turn leads to an excessive exchange of $\mathrm{Ca}^{2+}$ via the $\mathrm{Na}^{+} / \mathrm{Ca}^{2+}$ exchanger (15). The transport of $\mathrm{Ca}^{2+}$ between sarcoplasmic/endoplasmic reticulum and mitochondria enables cardiac excitation-contraction coupling (38). However, $\mathrm{Ca}^{2+}$ uptake by the sarcoplasmic reticulum $\mathrm{Ca}^{2+}$-ATPase is inhibited due to ATP depletion during ischemia. The rapid restoration of the $\Delta \Psi \mathrm{m}$ by the subsequent reperfusion drives the uptake of $\mathrm{Ca}^{2+}$ into the mitochondria via the mitochondrial calcium uniporter (15). Therefore, $\mathrm{Ca}^{2+}$ overload in the mitochondria is one of the hallmarks of IR injury of the heart (39). During the reperfusion phase, the accumulation of cytosolic $\mathrm{Ca}^{2+}$ activates calcineurin $(\mathrm{CaN})$ to dephosphorylate Drp1 at $\mathrm{Ser}^{637}$, initiating mitochondrial fission and cardiomyocyte apoptosis (40).

\section{Mitochondrial dynamics in response to IR stress}

Biogenesis. There are a large number of transcription factors and regulatory proteins to enhance mitochondrial biogenesis, such as nuclear respiratory factors 1 and 2, transcription factor A mitochondrial, peroxisome proliferator-activated receptors (PPARs), estrogen-related receptors, cAMP response element-binding proteins and Forkhead box-O. Transcription factors can be regulated by coactivators and corepressors, 
such as PPAR $\gamma$ coactivator-1 $\alpha$ (PGC-1 $\alpha)$ and nuclear receptor corepressor 1 . PGC- $1 \alpha$ can be activated by post translational modifications, such as through phosphorylation by AMP-activated protein kinase (AMPK) and deacetylation by Sirtuin 1 (Sirt1) (41). Under oxygen insufficiency, an energetic stress results in the downregulation of mitochondrial biogenesis by the hypoxia-inducible factor-mediated suppression of PGC-1 $\alpha$ (42).

Fission and fusion. Continuous mitochondrial fission and fusion enables a dynamic distribution of mitochondrial proteins and mtDNA through the cellular mitochondrial network (23). Mitochondrial fission involves division into two daughter mitochondria, one with a markedly higher mitochondrial membrane potential $(\Delta \Psi \mathrm{m})$ and fusion affinity than the other one. Subsequently, the active mitochondrion is further regenerated and the defective one is targeted for degradation via mitophagy to maintain mitochondrial homeostasis $(3,8,43)$. Fission and fusion proteins impact mitochondrial morphology and respiration, therefore, a balanced dynamic is essential to adjust mitochondrial metabolism to meet the energy demands in cardiomyocytes $(8,44,45)$. The alteration of proteins involved in mitochondrial fission and fusion is tightly associated with the pathophysiology of several cardiac diseases, such as IR stress and heart failure $(10,46)$.

The mitochondrial fission protein, Drp1, regulates mitochondrial dynamics on the mitochondrial outer membrane (47). Drp1 acts with Bcl-2 family proteins (such as the pro-apoptotic proteins Bax and Bak) to accelerate mitochondrial fragmentation and apoptosis (48). The phosphorylation at $\mathrm{Ser}^{637}$ residue of Drp1 by protein kinase A (PKA) inhibits its GTPase activity and its translocation to the mitochondria (49). Drp1 is enriched in the heart and its inhibition exhibits protective effects during myocardial IR by preventing excessive fission at the beginning of reperfusion $(36,40)$. The overexpression of the cardiac proto-oncogene Ser/Thr kinase, Pim-1, causes the downregulation of Drp1 and an increase in the levels of Ser ${ }^{637}$ phosphorylation, and also protects against cardiomyocyte ischemia (37). Drp1 can be reactivated via the dephosphorylation of $\mathrm{Ser}^{637}$ by the calmodulin-dependent kinase $\mathrm{CaN}$ (50). Under normoxic conditions, the actions of PKA and $\mathrm{CaN}$ on Drp1 are well regulated by mitochondrial scaffolding protein the A-kinase anchoring protein 1 (AKAP1) (50). However, in ischemic myocytes, AKAP1 is ubiquitinated by the hypoxia-induced E3-ubiquitin ligase Siah2 and rapidly degraded via the ubiquitin-proteasome system, which impairs PKA-mediated Drp1 inhibition and initiates mitochondrial fission and cardiomyocyte apoptosis $(39,50)$. In addition, cardiac IR injury may be reduced when the transient suppression of Drp1 has been achieved by small molecular disruptors or by shRNA/siRNA-mediated knockdown (36,40,51). Chronic Drp1 deficiency, such as the heterozygous knockout $\left(\operatorname{Drpl}^{+/}\right)$, results in enlarged infarct sizes after IR stress (52).

The mitochondrial fission factor (Mff) is important for Drp1 recruitment in mitochondria during the process of fission (53). The inhibition of Mff has been shown to enhance mitochondrial membrane potential $(\Delta \Psi \mathrm{m})$ and decrease apoptosis under hypoxia/reoxygenation (54).

The dynamin-related GTPases mitofusin (Mfn) 1 and 2 are responsible for the fusion of the mitochondrial outer membrane between two mitochondria (19), whereas, the optic atrophy protein 1 (OPA1) mediates mitochondrial inner membrane fusion via regulation of the cristae structure $(8,19)$. The downregulation of Mfn1 under conditions of ROS-induced stress during IR disturbs mitochondrial fusion and induces cardiomyocyte apoptosis (55). Conversely, Mfn2 is upregulated in response to the ROS burst during IR and induces the apoptosis of cardiomyocytes by inhibiting Akt (also known as protein kinase B) and activating caspase-9 (39). In the heart, Mfn2 is involved in the regulation of mitophagy, altering mitochondrial morphology and forming the ER/SR-mitochondrial contact sites (8). OPA1 plays a protective role in IR injury. $\mathrm{Opal}^{+-}$mice showed larger infarct sizes compared with wild type, and Opal overexpression results in decreased damage in cardiac function (17). Bax not only directly interacts with Mfn2 to physiologically regulate its assembly and distribution in the normal heart, but also co-localizes with Drp1 to facilitate mitochondrial fission during early apoptosis (39). The Bcl-2 family proteins, Bax, Bak and $\mathrm{Bcl} 2$-interacting protein 3 (BNIP3), promote OPA1 cleavage and mitochondrial cristae remodeling following reperfusion, which in turn exacerbate IR injury $(39,56)$.

Degradation and mitophagy. Mitochondrial dynamics facilitate mitochondrial quality control, which targets aberrant mitochondrial proteins or entirely damaged mitochondria for degradation to maintain myocardial mitochondrial homeostasis (57). The ubiquitin-proteasome system (UPS) and the autophagy-lysosomal system, termed mitophagy, are the major proteolytic pathways (58). The clearance of misfolded proteins usually occurs via UPS, whereas the entire damaged mitochondrion is degraded selectively by mitophagy (59). There are interactions and crosstalk between UPS and mitophagy in several regards, for example, the inhibition of proteasome induces the activation of mitophagy $(58,60)$.

Defective mitochondrial proteins are labeled with ubiquitin proteins by a series of E1, E2 and E3 enzymes and targeted into the proteasome for elimination (61). Impaired UPS proteolytic function has been observed in the heart tissue of patients with cardiomyopathy and heart failure (62); however, the enhancement of proteasome-dependent degradation plays a cardioprotective role against proteinopathy and IR injury in mouse models (63). However, the effects of proteasome inhibitors remain controversial, which could be both beneficial and detrimental on cardiac function during myocardial ischemia and reperfusion (64).

Autophagy is induced by the deprivation of oxygen and nutrition, and refers to the lysosomal degradation of unnecessary cellular components, such as protein aggregates, waste lipids and dysfunctional organelles (4). During selective mitochondrial autophagy (mitophagy), the dysfunctional mitochondria are tagged with polyubiquitin, embedded into autophagosomes and delivered to the lysosome for degradation $(23,65)$.

PTEN-induced putative kinase 1 (PINK1) and ubiquitin ligase Parkin-mediated mitophagy. Kinase PINK1 is a Ser/Thr protein kinase that phosphorylates ubiquitin, Parkin and the mitochondrial outer membrane protein Mfn2. The phosphorylation events activate Parkin to translocate from the cytosol to the mitochondria $(4,66)$. Parkin is an E3 ubiquitin 
ligase that polyubiquitinates the phosphorylated Mfn2 (67), in-turn promoting the binding of mitophagy proteins (sequestosome protein $\mathrm{p} 62 / \mathrm{SQSTM} 1$ and autophagy-related protein 8 mammalian homologue LC3) and the cargo sequestration within the autophagosome for the subsequent degradation in lysosomes $(4,66)$.

The loss of PINK1 has been shown to increase the myocardial infarct size and generate excess ROS during stimulated IR injury, whereas the overexpression of PINK1 reduces cardiac cell death following stimulated IR injury (68). On the one hand, Parkin-deficiency in the hearts of mice reduces mitophagy, resulting in the accumulation of dysfunctional mitochondria and enlarged infarcts following MI (69). However, excessive Parkin-mediated mitophagy also initiates the induction of apoptotic pathways in cardiac microcirculation endothelial cells and exacerbates microvascular injury, as well as cardiac reperfusion injury (70).

Receptor-mediated mitophagy. The mitophagy receptors, such as BNIP3, Nip3-like protein X (NIX) (also known as Bcl2-interacting protein 3 like) and FUN14 domain containing 1 (FUNDC1), localize to the mitochondrial outer membrane and serve a role in acute IR injury by regulating mitophagy $(4,23,62,65,66)$. For example, BNIP3 binds to stabilized PINK1, which facilitates the recruitment of Parkin on the mitochondrial outer membrane (71). NIX is ubiquitinated by Parkin, enhancing the recruitment of the autophagy receptor NBR1 and LC3-coated vesicles to the mitochondria (66). Under hypoxic conditions, the heart-enriched FUNDC1 interacts with LC3 to recruit autophagosomes to mitochondria and enhances mitophagy (72). The phosphorylation at $\mathrm{Ser}^{13}$ of FUNDC1 by casein kinase 2 (CK2) inhibits the interaction between FUNDC1 and Drp1, ultimately decreasing mitophagy (66). CK2 $\alpha$ has been reported to be upregulated during IR stress. As a consequence, FUNDC1 is deactivated and suppresses FUNDC1-mediated mitophagy (4). Ischemic preconditioning has been reported to induce extensive FUNCD1- or Parkin-mediated mitophagy in platelets and reduces secondary ischemic injury (22). In preclinical studies, myocardial conditioning interventions, including ischemic pre-, post-conditioning or remote pre-/per-conditioning, are widely used strategies to reduce infarct sizes following sustained IR. Several signaling pathways activated in response to conditioning stimuli end with the preservation of mitochondrial function (17).

Bcl2-associated athanogene (BAG) family molecular chaperone regulator 3 (BAG3)-mediated non-canonical mitophagy. BAG3 is highly expressed in cardiac muscles and functions as a chaperone to target misfolded proteins for lysosomal-mediated degradation (73). BAG3-Pro209Leu mutation causes the dysregulation of autophagy, which is associated with the rapid progression of restrictive cardiomyopathy in myofibrillary myopathies (74).

Mitochondrial heterogeneity and subpopulations. In neonatal cardiomyocytes, the mitochondria are distributed throughout the cytosol; however, in the adult cardiac cells, the mitochondria are small, round, hypodynamic and relatively static. In particular, they are heterogeneous and are clustered into sub-sarcolemmal mitochondria (SSM), inter-myofibril mitochondria (IFM) and perinuclear mitochondria subsets with distinct morphologies, localizations and functions $(8,17,75)$. These three mitochondrial subpopulations also possess different sensitivities to pathological processes, such as IR injury. For example, SSM exhibit greater oxidative damage and increased reduction in complex I-mediated respiration than IFM, whereas IFM consume less oxygen than SSM in complex II following IR $(17,76)$. Therefore, the mitochondrial defects in ischemic cells are in a heterogeneously distributed manner amongst distinct subpopulations (76,77). A significant heterogeneity of redox states, $\mathrm{Ca}^{2+}$ and $\Delta \Psi \mathrm{m}$ has also been shown in myocardial cells following IR injury (76).

\section{Current developments in mitochondria-targeted agents with cardioprotective effects against IR injury}

The defective mitochondria display several different types of abnormalities and disturbances in metabolism and morphology, which are often reciprocally coupled (41) and contribute to acute, irreversible cell death and potentially, MI (77). The prevalence of ischemic heart disease is statistically the highest in Europe and North America. An estimated 8.4 million American adults ( $\geq 20$ years old) suffered an MI between 2013 and 2016. The mortality related to MI in 2017 was 0.1 million in the USA. Additionally, ischemic heart diseases have a notable financial impact. For example, the arithmetic mean cost for each discharge due to acute coronary syndrome (ACS) was $\$ 63,578$ in 2009. Productivity losses connected to ACS were $\sim \$ 8,000$ (short-term) and $\$ 50,000$ (long-term) per disability claim between 2007 and 2010 according to medical, pharmacy and disability insurance data (78). Therefore, drugs designed to directly act on the mitochondria for IR injury therapy would have a substantial impact on both medical and societal costs.

With a growing understanding of the molecular mechanisms of IR injury, several mitochondrion-targeted agents are under research and development, which are planned to be used for cardiac IR injury treatment (Table I). These agents have been designed to improve mitochondrial function via different underlying strategies, for example: i) Decreasing the accumulation of succinate during ischemia; ii) reducing succinate oxidation following reperfusion; iii) inhibiting RET at complex I; iv) counteracting the superoxide production; v) reducing ROS damage; vi) increasing NAD ${ }^{+}$levels; vii) blocking the mPTP; viii) maintaining $\mathrm{Ca}^{2+}$ homeostasis; and ix) regulating mitochondrial dynamics and quality control.

Inhibitors of respiratory complex II (SDH). Malonate is a competitive inhibitor of SDH, reducing succinate accumulation and oxidation during myocardial IR injury. Dimethyl malonate is a cell membrane-permeable form of malonate and its protective effect against IR injury have been investigated in purified mitochondria, mouse hearts, and in murine and rabbit models of stroke $(14,79,80)$.

Chloramphenicol succinate (CAPS) is a prodrug of chloramphenicol (CAP), which is already approved for clinical use as a human antibiotic, but with a risk of hemotoxicity, including reversible and irreversible anemia. CAP-induced reversible anemia with reticulocytopenia, often in conjunction with leucopenia and thrombocytopenia, develops with the increasing dose of CAP during treatment. Daily dose levels 
Table I. Research and development status of the current mitochondria-targeted agents with cardioprotective effects against ischemia-reperfusion injury.

Development stage

Mitochondria-targeted agent

Discovery: Natural source, highthroughput screening and drug design

Pre-clinical: Tested in cells, isolated tissues and animals to determine efficacy, toxicity and pharmacokinetic properties

Clinical trials: Tested in humans

Phase I

Phase II

Phase III

Marketing, approved

\section{MitoGSH (96)}

Dimethyl malonate (80), chloramphenicol succinate (83), MitoSNO (84), amobarbital (85), S1QELs (86), SkQ (88), Euk-8 (97), CMX-2043 ( $\alpha$-lipoic acid) (98), NIM811 (105), sanglifehrin A (106), 5-aminoimidazole-4-carboxamide ribonucleotide $(115,116)$, resveratrol $(123)$, mitochondrial division inhibitor-1 (125), P110 (51), rapamycin (126), miR-499 (127)

Bendavia (NCT01572909), nicotinamide mononucleotide (UMIN000021309) MitoQ (NCT00433108), rosiglitazone (NCT00064727)

Coenzynme $\mathrm{Q}_{10}$ (ISRCTN94506234), cyclosporine A (NCT01650662), bezafibrate (https://www.acc.org/Latest-in-Cardiology/Clinical-Trials/2013/04/13/21/02/BIP), pioglitazone (NCT00091949), metformin (NCT01217307)

None of CAPS between $500-3,500 \mathrm{mg} / \mathrm{kg}$ have been observed to induce significant reversible myelotoxicity in different mouse strains. CAP-induced irreversible anemia is a non-dose-related aplastic anemia with a severe pancytopenia, which is fatal and unpredictable with an incidence of 20-40 cases per million following therapies (81). CAPS can be hydrolyzed by esterase or oxidized by SDH into succinate and CAP, indicating that CAPS may be a competitive substrate of SDH. The active metabolite, CAP, in turn inhibits SDH reductive function, underlying the potential myelotoxicity induced by CAPS (82). The cardioprotective effect of CAPS has been shown in a pig model of MI, where reduced infarct sizes were observed following pre-ischemic in vivo administration of CAPS, as well as in the delayed treatment, where CAPS was administered prior to reperfusion. CAPS may also induce autophagy, indicated by the upregulated specific autophagy markers Beclin-1 and microtubule-associated protein light chain (LC3-II). However, the specific molecular mechanisms underlying its action remain unclear (83).

Inhibitors of respiratory complex I. MitoSNO is a mitochondria-targeted S-nitrosating agent, temporarily inhibiting complex I by reversible S-nitrosation of ND3 $\mathrm{Cys}^{39}$ residue during ischemia. The capability of MitoSNO as a potential drug has been assessed through incubation of mitochondria from rat and mouse hearts, ex vivo heart treatment and in vivo administration in mice models of MI (84).

Amobarbital is also a rapidly reversible inhibitor of complex I, which can improve mitochondrial function and protect cell survival during cardiac IR injury possibly via reducing the release of apoptosis inducing factor from the mitochondrial intermembrane space into cytosol. The protective effects of amobarbital against cardiac IR damage has been assessed in buffer perfused rats and Harlequin (Hq) mouse hearts (85).

Following high-throughput chemical screening and validation, performed by Brand et al (86), two families of the cell-permeant compounds synthesized from commercial companies were identified as effective and selective suppressors of ubiquinone (Q)-binding site of respiratory complex I (site $\mathrm{I}_{\mathrm{Q}}$ ). Suppressors of site $\mathrm{I}_{\mathrm{Q}}$ electron leak (S1QELs), S1QELs 1.1-1.6 and S1QELs 2.2-2.4, can inhibit ROS production at complex I during reverse electron transport without altering basal respiration or the resting OXPHOS. S1QEL1.1 is an outstanding candidate, which strongly suppresses tunicamycin-triggered endoplasmic reticulum stress and caspase signaling pathways that leads to apoptosis in cardiomyocyte $\mathrm{H} 9 \mathrm{c} 2$ cells and protects Langendorff-perfused mouse heart models of IR injury from endogenous oxidative damage, including enhancement of cardiac function in post-ischemic recovery and the decrease in infarct size. This highlights S1QEL1.1 as a novel therapeutic agent which can be used at the time of reperfusion. The suppression of ROS generation and oxidative stress signaling by S1QELs have also been assessed in isolated rat skeletal muscle mitochondria, 293 cells, primary astrocytes and live Drosophila melanogaster (86).

Mitochondria-permeable or -targeted antioxidants. Coenzyme $\mathrm{Q}_{10}\left(\mathrm{CoQ}_{10}\right)$ is an endogenous lipid, with an oxidized-form (ubiquinone) and fully reduced-form (ubiquinol), which is the most common form of CoQ in humans. In addition to its primary role in electron- and proton-transport in mitochondria for production of ATP (87), $\mathrm{CoQ}_{10}$ is also a mitochondria-permeable antioxidant (88). $\mathrm{CoQ}_{10}$ deficiency is associated with several diseases, such as cardiovascular diseases, muscular dystrophy and neurodegenerative disorders (87). Administration of $\mathrm{CoQ}_{10}$ as a supplement along with standard clinical therapy shows positive effects in patients with hypertension (87) and heart failure (89). Higher plasma $\mathrm{CoQ}_{10}$ concentrations in patients with $\mathrm{MI}$ lead to improved left ventricular function. An intravenous $\mathrm{CoQ}_{10}$ injection in rat models of coronary artery blockage has been shown to reduce cardiomyocyte necrosis and limit post-infarction hypertrophy of the left ventricle (87). A significant improvement in patients with chronic heart failure was shown in the Q-SYMBIO study (a randomized double-blind trial of 420 international patients) (90). 
MitoQ is a bioactive ubiquinone covalent compound linked with a lipophilic cation triphenylphosphonium (TPP), which is reduced into the antioxidant ubiquinol in the mitochondria, and protects against oxidative injury. MitoQ is a promising mitochondria-targeted antioxidant, which has shown positive results in an intravenous and oral toxicity study in mice, in long-term oral administration studies in mice and rats, and in human Phase I and II trials (no significant difference in the NCT00329056 study and a potential benefit in the NCT00433108 study) $(91,92)$.

10-(6'-Plastoquinonyl) decyltriphenyl-phosphonium (SkQ) is a lipophilic cation attached to a plastoquinone, which is a mitochondria-targeted antioxidant that protects cells from age-related diseases and ROS-burst-mediated acute diseases, such as IR. SkQ has not yet been approved by the US Food and Drug Administration as the safety and the clinical application of SkQ requires further study (88).

Bendavia (also known as SS31 and MTP-131) is a Szeto-Schiller (SS) peptide that can penetrate the mitochondria and ameliorate IR injury by interacting with the phospholipid cardiolipin and protecting mitochondrial cristae membranes (93). Positive results have been indicated in animal studies $(93,94)$; however, Bendavia was unsuccessful in the EMBRACE STEMI study (Phase 2a trial). Therefore, MTP-131 is considered a potential pharmacological compound that protects mitochondria, but has not yet successfully translated into clinical use for the patients with ST-elevation MI (95).

MitoGSH is a group of synthetic forms of reduced glutathione (GSH) tagged with SS peptides or TPP ions that can target the mitochondria. MitoGSH is a class of novel drugs that may be highly valuable compounds for treatment of heart diseases by effectively restoring mitochondrial GSH levels. However, the limitations of TPP and SS peptides should be taken into consideration (96).

Euk-8 is a SOD/catalase mimetic antioxidant that is capable of potently scavenging oxyradicals. The positive cardiac-protective effects of Euk- 8 include the reduction of both oxidative stress and pressure overload-triggered heart failure in the X-linked Hq mutant and WT mice (97).

$\alpha$-lipoic acid is an important mitochondria-permeable antioxidant (88). $\alpha$-lipoic acid is a natural dithiol that exerts protective effects against IR injury in several organs (98) and is used as a treatment of diabetic neuropathy and eye-disorders clinically, but has several side effects (88). CMX-2043 [N-((R)-1,2-dithiolane-3-pentanoyl)-L-glutamyl-L-alanine] is a novel analogue of $\alpha$-lipoic acid, which has been shown to achieve efficacious reduction of infarct damage in a rat model of myocardial IR injury (98).

Precursors of $\mathrm{NAD}^{+}$. Nicotinamide riboside (NR) and nicotinamide mononucleotide (NMN) are the natural precursors of $\mathrm{NAD}^{+}$. The supplementation of NR or NMN can increase cellular $\mathrm{NAD}^{+}$levels, without any noticeable side-effects $(99,100)$. Bioactive NMN is synthesized from nicotinamide, a phosphate group and a ribonucleoside by nicotinamide phosphoribosyltransferase (100). Both NR and NMN have been shown to exert beneficial effects on cardiovascular functions under IR conditions. The administration of NMN has been shown to exhibit timing-dependent patterns on the reduction of infarct size (before ischemia or before reperfusion) (100). Several clinical studies regarding the safety and effects of NR on human physiology are currently underway in Europe and the USA (NCT03432871, NCT03423342 and NCT02812238). NMN is available on the market as a dietary supplement. A Phase I human clinical study for NMN has commenced in Japan and has evaluated the safety and the bioavailability of NMN in humans, with the aim of developing NMN into an anti-aging nutraceutical $(99,101)$.

Inhibitors of $m P T P$. Cyclosporine A (CsA) is widely used as an immunosuppressive drug following organ transplantation and autoimmune disorders clinically, due to its ability to suppress the transcription of cytokines in activated $\mathrm{T}$ cells via blocking the CaN/nuclear factor of activated $\mathrm{T}$ cells pathway $(95,102)$. However, CsA is not only an inhibitor of cytosolic $\mathrm{CaN}$, but also an inhibitor of mPTP, binding to mitochondrial cis-trans prolyl isomerase cyclophilin $\mathrm{D}$ (CyD) (95). CyD is a positive regulator of $\mathrm{mPTP}$ with a chaperone localized in the mitochondrial matrix $(3,8)$. The cardioprotective effects of CsA have been assessed in several species of animals using reperfused MI models, with inconsistent results in reduction of infarct size (95). Patients with ST-elevation MI (STEMI) administered CsA have exhibited promising results in a Phase II trial; however, CsA was unsuccessful in the Phase III CIRCUS and CYCLE trials $(95,103,104)$.

NIM811 is a CsA derivative without immunosuppressive capabilities, which can also specifically inhibit mPTP opening in the mitochondria from the reperfused myocardium (95). Adult male New Zealand white rabbits injected with NIM811 at the time of reperfusion have been shown to exhibit preserved OXPHOS in the heart mitochondria, reduced infarct sizes and reduced myocardial damage (105).

Sanglifehrin A (SfA) is a specific inhibitor of cyclophilin D, similar to $\mathrm{CsA}$, although it cannot suppress $\mathrm{CaN}$ activity. The specific inhibition of CyD by SfA protects against cardiac IR injury by limiting mPTP opening $(3,106)$. The beneficial effects of SfA have been examined in MI female rat hearts. Significantly improved cardiac function was only shown in reperfused hearts treated with one intravenous bolus of SfA in the acute phase (2 days) of post-MI remodeling (106).

PPAR agonists for the activation of mitochondrial biogenesis. Fibrates have been discovered to activate PPARs, particularly $\operatorname{PPAR} \alpha$, which regulate glucose and lipid metabolism in the heart. Fibrates have been widely used as a class of hypolipidemic drugs for treatment of heart attacks and strokes (107,108). Bezafibrate (marketed as Bezalip) is a fibrate drug widely used for the treatment of metabolic syndrome and hyperlipidemias. Bezafibrate is an agonist of PPAR $\alpha$ and partially activates PPAR $\gamma$ and PPAR $\delta$, upregulating mitochondrial biogenesis by increasing PGC- $1 \alpha$ expression (109). A small clinical trial including patients with acute STEMI and hyperfibrinogenemia showed bezafibrate treatment resulted in lower fibrinogen levels and lower frequency of major cardiovascular events compared with conventional therapy (110). The clinical trial Bezafibrate Infarction Prevention (BIP) study with 16 years of follow-up has shown that bezafibrate treatment reduces the risk of cardiac death and the non-fatal MI as well as mortality in patients with coronary arterial diseases (107). 
Thiazolidinediones (TZDs) are structurally and pharmacologically similar to fibrates and more specifically act on PPAR $\gamma$. TZD (also known as glitazone) is a class of hypoglycemic drugs used for treatment of type 2 diabetes clinically and protects against cardiovascular diseases. Pioglitazone and rosiglitazone are TZDs, which are also PPAR $\gamma$ agonists and are used as anti-diabetic drugs (108). With the risk of bias and uncertain adverse events, pioglitazone was shown to also reduce non-fatal MI and fatal or non-fatal stroke in four clinical studies (IRIS, NCT00091949; J-SPIRIT, UMIN000013499; Kernan et al (111); and PROactive, NCT00174993) (112). Rosiglitazone has exhibited opposite results in pre-clinical and clinical trials. Rosiglitazone administration to pig and rat with IR-affected hearts has been shown to significantly decrease the infarct size, but without improvement of cardiac mitochondrial function (113). From the data pooled from 42 clinical studies, a significant increase in MI and severe adverse cardiovascular causes were observed with the use of rosiglitazone for patients with type 2 diabetes (114). Only one phase II clinical trial (NCT00064727) has been completed to investigate the therapeutic efficiency of rosiglitazone in patients with congestive heart failure (107).

Activators of mitochondrial biogenesis. 5-Aminoimidazole4-carboxamide ribonucleotide (AICAR) is an AMPK agonist, activating PGC-1 $\alpha$ by mimicking AMP (109). Serum-starved rat cardiomyocytes stimulated with AICAR have exhibited the full activation of AMPK downstream targets, such as Sirt1 and PGC-1 $\alpha$, which may contribute to mitochondrial homeostasis and cardioprotective effects against ischemic stress (115). Dogs treated with AICAR during cardiopulmonary bypass surgery followed by reperfusion have exhibited an improved recovery of myocardial glucose uptake and utilization (116).

Metformin is also an AMPK activator which exerts protective effects against cardiac ischemia, MI and heart failure. Additionally, metformin is a hypoglycemic drug widely used for the treatment of diabetes. Metformin has been proposed to reduce myocardial IR injury via increasing PGC- $1 \alpha$ levels and mitochondrial biogenesis (117). Even though both preclinical and clinical studies (such as the DIGAMI 2 trial) have suggested the cardioprotective effects of metformin against IR injury (118-121), 4 months of metformin treatment in the GIPS-III trial did not result in improved left ventricular function in patients without diabetes presenting with STEMI. The 2-year follow-up results of the GIPS-III trail also revealed no beneficial long-term effects (122).

Resveratrol is a natural polyphenol (stilbenoid) found in red wine, that functions as a Sirt1 and PGC- $1 \alpha$ activator, and an inhibitor of mitochondrial ATPase (109). The protective effects of resveratrol have been shown in animal models of cardiovascular, neurodegenerative and metabolic disorders. Five clinical trials of resveratrol for treatment of pre-diabetes and pulmonary diseases are currently in progress (NCT02502253; NCT02565979; NCT03762096; NCT02245932; NCT03819517). However, resveratrol has limitations in human therapeutic application due to its low potency and poor pharmacokinetics. Resveratrol and several other polyphenols are often used as dietary antioxidants (123).

Inhibitors of mitochondrial fission. Mitochondrial division inhibitor-1 (Mdivi-1) is a derivative of quinazolinone, which can inhibit mitochondrial fission by decreasing Drp1 activity, thereby reducing short term ischemic stress and myocardial IR injury. Mdivi-1 has been shown to exert cardioprotective effects in pre-treated cardiomyocytes (HL-1 cells), primary isolated neonatal mice ventricular myocytes, hearts from Langendorff perfusion in a rat model of IR, a murine coronary artery ligation model and a pressure overload mouse model with induced heart failure $(40,124,125)$.

P110 is a small peptide (7-amino acids) which blocks the contact between Drp1 and mitochondrial fission 1 protein, ameliorating MI and long-term heart remodeling. P110 peptide has been demonstrated to inhibit excessive mitochondrial fission in primary neonatal rat cardiomyocytes, decreasing the infarct size and improving cardiac function in an ex vivo Langendorff rat heart model and an in vivo rat model of MI (51).

Regulator of mitophagy. Rapamycin has been found to promote mitophagy by inhibiting the mammalian target of rapamycin (mTOR) pathway and reducing infarct size in MI and cardiomyocyte apoptosis. As an allosteric inhibitor of mTOR complex I (mTORC1), rapamycin plays a role in the suppression of cell growth and division, and has been used as an immunosuppressant drug, or for the treatment of cancer and in-stent restenosis in clinical practice. Rapamycin has also been shown to reduce cardiomyocyte hypertrophy in a mouse model of chronic ischemic injury and to inhibit cardiomyocyte apoptosis in vitro (angiotensin II-induced myocyte apoptosis model) and in vivo (MI-induced chronic heart failure rat model) (126).

MicroRNA (miRNA/miR) regulation. miR-499 is an intronic miRNA identified to be involved in inhibiting apoptosis and MI induced by ischemia. miR-499 suppresses the expression of $\mathrm{CaN}$ and reduces cell apoptosis following cardiac ischemia, protecting against the MI. miR-499 transgenic mice were previously created, and when they were intravenously injected with the antagomir of miR-499 following IR induction via surgery, they were used for the in vivo evaluation of cardiac function, and hearts harvested from these mice were used for histological and western blot analysis (127).

\section{Future perspectives}

Limiting early mtROS production in IR injury. Superoxide production driven by succinate oxidation upon reperfusion through RET at complex I in the mitochondrial respiratory chain is considered the major source of ROS in IR injury (5). In vivo experiments have indicated that the succinate accumulated during ischemia undergoes rapid oxidation and returns to normoxic levels within 5 min following reperfusion (14). Therefore, it could be an effective therapeutic strategy for the treatment of IR injury by inhibiting RET at complex I for at least $5 \mathrm{~min}$, until the succinate is no longer functional as an electron sink (Fig. 2).

The electron transport chain complex I undergoes a structural change, which is termed 'active/deactive transition' during IR (128). $\mathrm{Cys}^{39}$ in complex I is the critical switch of this conformational transition (Fig. 2), which is exposed in the deactive state of complex I under ischemic conditions and 


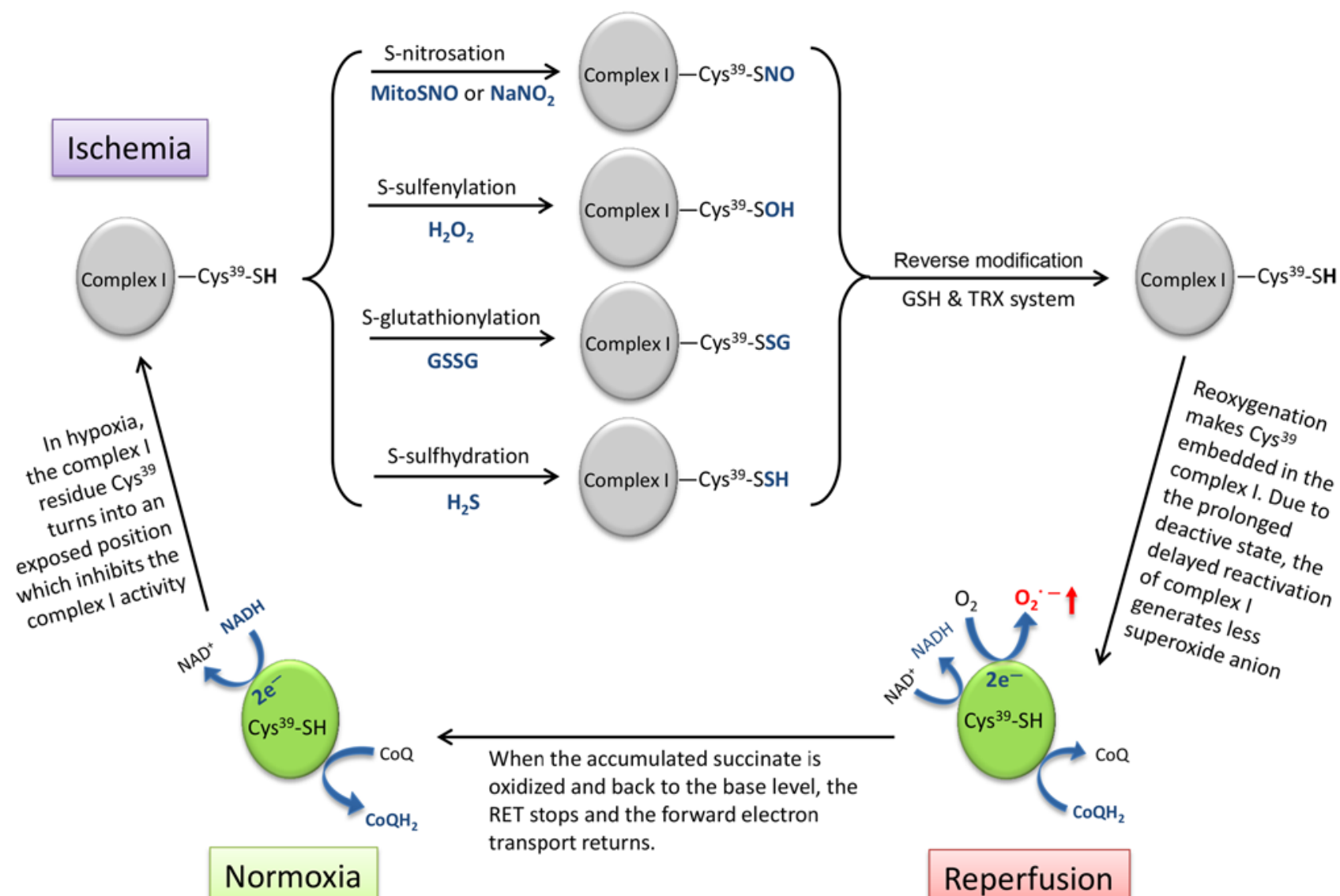

Figure 2. Schematic diagram showing the strategy of using the characteristics of complex I active/deactive transition to reduce ROS production upon reperfusion by temporarily locking complex I in the deactive state with reversible modifications on residue Cys ${ }^{39}$. ROS, reactive oxygen species; GSH, glutathione; TRX, thioredoxin; GSSG, glutathione disulfide.

embeds in the reactivated complex I upon reperfusion $(5,129)$. Several thiol-reactive agents can be used to lock complex I in the deactive state by thiol modification on $\mathrm{Cys}^{39}$, including S-nitrosation (-SH into -SNO) by S-nitrosothiols (MitoSNO) or sodium nitrite $\left(\mathrm{NaNO}_{2}\right)(84,130)$, S-sulfenylation (-SH into -SOH) by $\mathrm{H}_{2} \mathrm{O}_{2}$ (131), S-glutathionylation (-SH into -SSG) by glutathione disulfide (GSSG) (132) and S-sulfhydration/persulfidation (-SH into -SSH) by hydrogen sulfide $\left(\mathrm{H}_{2} \mathrm{~S}\right)(133)$. These post-translational modifications can later be reversed by the internal mitochondrial GSH and thioredoxin systems (84).

However, the appropriate onset and duration of administration of thiol-reactive agents remains uncertain, otherwise this intervention based on the structural interconversion of complex I may be an ideal therapeutic method for preventing the burst of ROS production via RET and to return complex I activity in time.

Mitochondrial quality control. A poor quality of mitochondrial network generates and accumulates excess ROS (35). The burst of mtROS results in a series of downstream oxidative damages in cardiomyocytes after acute MI or during cardiac senescence $(134,135)$, which may lead to sudden cardiac death or heart failure (136).

Mitophagy plays an important role in maintaining mitochondrial quality control, particularly in adult cardiomyocytes $(43,137)$. However, cardiac mitophagy plays both beneficial and detrimental roles in response to IR stress $(22,66,138)$. On the one hand, the clearance of the defective mitochondria via mitophagy may reduce the risk of ROS release and cell apoptosis, which exerts protective effects by decreasing myocardial oxidative stress and infarct size following MI $(23,137,138)$. During IR, additional mitochondrial mass causes excess oxygen consumption and ATP depletion, which exacerbates the energetic stress. When energy is insufficient, active mitophagy can enhance cell survival $(19,23)$. However, excessive mitophagy causes a drastic loss of mitochondrial mass and ATP supply in cardiomyocytes, leading to cell death and heart failure $(54,66)$. When mitophagy is insufficient, the accumulation of damaged mitochondria and mtROS weakens the adaptiveness of cardiomyocytes towards cellular stress and apoptosis $(4,66)$. Therefore, intervention with properly modulated mitophagy can serve as an ideal, yet difficult to achieve, therapeutic method for cardiac IR stress and other diseases $(19,66,138)$. Both mitophagy and mitochondrial biogenesis are upregulated following heart surgery (139). Thus, it is essential to maintain a balance between mitochondrial degradation and biogenesis to maintain mitochondrial homeostasis and myocardial protection under stress $(138,140)$.

Attention must be paid to mitophagy, which is a complex process and intersects with several other cellular events. Some mediators in mitophagy signaling pathways, such as mTOR and AMPK, also play roles in mitochondrial biogenesis $(19,66)$, intrinsic fatty acid metabolism (141), glucose-modulated 
amino acid sensing and utilization (142), endothelium-dependent vasodilation in vessels (143), vascular smooth muscle cell proliferation and migration (144), suggesting that the manipulation of these may cause a series of unexpected side-effects.

Gap between pre-clinical studies and clinical practice of putative infarct-sparing drugs. As discussed above in Chapter 5 entitled 'Current developments in mitochondria-targeted agents with cardioprotective effects against IR injury', even though several agents have shown promising cardioprotective effects in pre-clinical studies, notable clinical trials in the past have highlighted the limitations or translational failures. The administration of certain autacoids, such as adenosine, bradykinin and opioids, can mimic the cardioprotective state of ischemic pre-conditioning, triggering several signaling pathways and finally inhibiting the formation of mPTP. As adjunctive therapies, a common limitation of these pre-conditional mimetics is the prerequisite administration before lethal ischemia, which makes them suitable for the planned cardiac surgeries but not effective in the clinical practice of acute MI. Two large-scale clinical trials, AMISTAD I and II, which evaluated the effects of adenosine during acute STEMI therapy, exhibited restrictive infarct size-reduction and a number of adverse events, such as hypotension, bradycardia, heart block and ventricular tachycardia, especially in the adenosine patients with nonanterior MI (145).

Bradykinin, as the vasodilatory peptide, plays a role in decreasing blood pressure and increasing vascular permeability (146). Bradykinin signaling deficiency may be responsible for the vascular contraction and the high epithelial $\mathrm{Na}^{+}$reabsorption observed in patients with hypertension and cardiovascular diseases. Bradykinin signaling has also been demonstrated to reduce inflammation and endothelial apoptosis in animal models of MI and cardiac IR injury (147). However, an increasing bradykinin concentration either inherited or acquired, which is a main mediator of hyperpermeability of capillaries and accumulation of local edema fluid, results in a rare disease termed bradykinin-mediated angioedema $(146,148)$.

Opioids, such as morphine have been widely and frequently used as analgesics for the patients with STEMI (149). First of all, STEMI patients often have intense chest pain and anxiety in the acute phase of the treatment. As a traditional analgesia, opioids are effective in symptomatic relief. In addition, the reduction of pain and stress also results in vasodilation, an increase in blood flow, a decrease in arterial blood pressure and heart rate, equilibrating the supply and demand of oxygen and nutrients in cardiomyocytes $(149,150)$. However, increased severe side-effects of opioids in STEMI patients have been revealed in recent clinical studies. For example, the low myocardial contractility, hypotension and fainting due to morphine-induced peripheral vasodilation are the main hemodynamic adverse effects for the patients with ventricular failure. A long-term use of opioids raises the risk for respiratory and gastrointestinal adverse effects, such as suppression of breathing, gut motility and absorption (150). Therefore, opioid administration may disrupt the absorption of certain essential oral medicaments, such as platelet inhibitors, and consequently delay the action of antiplatelet agents and prolong the platelet reactivity in the treatment of STEMI (149).
One of the major reasons for the limited clinical success is incomplete or insufficient pre-clinical investigation that does not offer accurate, reproducible and systematic examination of results from multiple animal models before large-scale clinical trials. The multicenter CAESAR (Consortium for PreclinicAl AssESsment of CARdioprotective Therapies) initiative supported by the National Heart Lung and Blood Institute was created to improve translation of cardioprotective therapies into clinical use (151). CAESAR promotes integrated, standardized, solid and rigorous preclinical evaluations to examine potential drugs or combined therapies in centralized laboratories.

\section{Conclusions}

In conclusion, $\mathrm{MI}$ is one of the leading causes of mortality and economic burden on health systems. Cardiac IR injury following MI is due to the oxidative damage from mtROS, which leads to severe mitochondrial damage and cardiomyocyte death. Therefore, the mitochondria are strongly recommended to be considered as a therapeutic target to prevent cardiac IR injury. Several promising mitochondria-targeted agents are under research and development with significant focus on protecting against cardiac IR injury by counteracting the changes in mitochondrial metabolism and/or morphology during IR. However, none of these have exhibited sufficient efficacy and safety for clinical use. For future studies, some theoretical and practical points are suggested to be taken into consideration: Limiting ROS production by the reversible and temporary interference on the complex I structure is an interesting novel potential strategy. In addition, the side-effects and limitations of drug use should be thoroughly examined. A beneficial short-term outcome could lead to undesirable long-term outcomes over time, such as mitophagy, which is a double-edged sword for the mitochondrial quality and cell survival. Finally, an innovative multicenter approach is suggested, such as that advocated by CAESAR, in order to improve the translation of drugs from the bench to the bedside.

\section{Acknowledgements}

Not applicable.

\section{Funding}

The present study was funded by grants from the National Natural Science Foundation of China (nos. 81702785 and 81802822) and supported by the postdoctoral programme of Qingdao University.

\section{Availability of data and materials}

Not applicable.

\section{Authors' contributions}

WM conceived the idea for the review, drafted and revised the manuscript. DM contributed to the revision, the provision of some of the data and databases, and also contributed to the proofreading and language correction of the manuscript. 
WM and XA performed the literature search and prepared the figures. YL critically discussed and revised the manuscript, and provided some updates on the literature and clinical trials. All authors read and approved the final manuscript.

\section{Ethics approval and consent to participate}

Not applicable.

\section{Patient consent for publication}

Not applicable.

\section{Competing interests}

The authors declare that they have no competing interests.

\section{References}

1. Carlucci A, Adornetto A, Scorziello A, Viggiano D, Foca M Cuomo O, Annunziato L, Gottesman $M$ and Feliciello A: Proteolysis of AKAP121 regulates mitochondrial activity during cellular hypoxia and brain ischaemia. EMBO J 27: 1073-1084, 2008.

2. Haidarali S, Patil CR, Ojha S, Mohanraj R, Arya DS and Goyal SN: Targeting apoptotic pathways in myocardial infarction: Attenuated by phytochemicals. Cardiovasc Hematol Agents Med Chem 12: 72-85, 2014

3. Kuznetsov AV, Javadov S, Margreiter R, Grimm M, Hagenbuchner J and Ausserlechner MJ: The role of mitochondria in the mechanisms of cardiac ischemia-reperfusion injury. Antioxidants (Basel) 8: 454, 2019.

4. Yang M, Linn BS, Zhang Y and Ren J: Mitophagy and mitochondrial integrity in cardiac ischemia-reperfusion injury. Biochim Biophys Acta Mol Basis Dis 1865: 2293-2302, 2019.

5. Chouchani ET, Pell VR, James AM, Work LM, Saeb-Parsy K, Frezza C, Krieg T and Murphy MP: A unifying mechanism for mitochondrial superoxide production during ischemia-reperfusion injury. Cell Metab 23: 254-263, 2016.

6. Lesnefsky EJ, Chen Q, Tandler B and Hoppel CL: Mitochondrial dysfunction and myocardial ischemia-reperfusion: Implications for novel therapies. Annu Rev Pharmacol Toxicol 57: 535-565, 2017.

7. Eltzschig $\mathrm{HK}$ and Eckle T: Ischemia and reperfusion-from mechanism to translation. Nat Med 17: 1391-1401, 2011.

8. Wang W, Fernandez-Sanz C and Sheu SS: Regulation of mitochondrial bioenergetics by the non-canonical roles of mitochondrial dynamics proteins in the heart. Biochim Biophys Acta Mol Basis Dis 1864: 1991-2001, 2018

9. Spinelli JB and Haigis MC: The multifaceted contributions of mitochondria to cellular metabolism. Nat Cell Biol 20: 745-754 2018.

10. Niemann B, Schwarzer M and Rohrbach S: Heart and mitochondria: Pathophysiology and implications for cardiac surgeons Thorac Cardiovasc Surg 66: 11-19, 2018.

11. Bender DA: Oxidative phosphorylation. In: Encyclopedia of food sciences and nutrition. Caballero B (ed). 2nd edition. Academic Press, Oxford, pp4295-4301, 2003.

12. Murphy MP: How mitochondria produce reactive oxygen species. Biochem J 417: 1-13, 2009.

13. Guzy RD, Sharma B, Bell E, Chandel NS and Schumacker PT: Loss of the SdhB, but Not the SdhA, subunit of complex II triggers reactive oxygen species-dependent hypoxia-inducible factor activation and tumorigenesis. Mol Cell Biol 28: 718-731, 2008.

14. Chouchani ET, Pell VR, Gaude E, Aksentijevic D, Sundier SY, Robb EL, Logan A, Nadtochiy SM, Ord ENJ, Smith AC, et al: Ischaemic accumulation of succinate controls reperfusion injury through mitochondrial ROS. Nature 515: 431-435, 2014.

15. Pell VR, Chouchani ET, Frezza C, Murphy MP and Krieg T: Succinate metabolism: A new therapeutic target for myocardial reperfusion injury. Cardiovasc Res 111: 134-141, 2016.

16. Bugger $\mathrm{H}$ and Pfeil $\mathrm{K}$ : Mitochondrial ROS in myocardial ischemia reperfusion and remodeling. Biochim Biophys Acta Mol Basis Dis 1866: 165768, 2020.
17. Boengler K, Lochnit G and Schulz R: Mitochondria 'THE' target of myocardial conditioning. Am J Physiol Heart Circ Physiol 315: H1215-H1231, 2018.

18. Lin MT and Beal MF: Mitochondrial dysfunction and oxidative stress in neurodegenerative diseases. Nature 443: 787-795, 2006

19. Marzetti E, Csiszar A, Dutta D, Balagopal G, Calvani R and Leeuwenburgh C: Role of mitochondrial dysfunction and altered autophagy in cardiovascular aging and disease: From mechanisms to therapeutics. Am J Physiol Heart Circ Physiol 305: H459-H476, 2013.

20. Nordberg J and Arner ES: Reactive oxygen species, antioxidants, and the mammalian thioredoxin system. Free Radic Biol Med 31: $1287-1312,2001$

21. Judge $S$ and Leeuwenburgh C: Cardiac mitochondrial bioenergetics, oxidative stress, and aging. Am J Physiol Cell Physiol 292: C1983-C1992, 2007.

22. Zhang W, Chen C, Wang J, Liu L, He Y and Chen Q: Mitophagy in cardiomyocytes and in platelets: A major mechanism of cardioprotection against ischemia/reperfusion injury. Physiology (Bethesda) 33: 86-98, 2018.

23. Tahrir FG, Langford D, Amini S, Mohseni Ahooyi T and Khalili K: Mitochondrial quality control in cardiac cells: Mechanisms and role in cardiac cell injury and disease. J Cell Physiol 234: 8122-8133, 2019

24. Paradies G, Petrosillo G, Paradies V and Ruggiero FM: Role of cardiolipin peroxidation and $\mathrm{Ca} 2+$ in mitochondrial dysfunction and disease. Cell Calcium 45: 643-650, 2009.

25. Kay L, Nicolay K, Wieringa B, Saks V and Wallimann T: Direct evidence for the control of mitochondrial respiration by mitochondrial creatine kinase in oxidative muscle cells in situ. J Biol Chem 275: 6937-6944, 2000.

26. Dolder M, Wendt S and Wallimann T: Mitochondrial creatine kinase in contact sites: Interaction with porin and adenine nucleotide translocase, role in permeability transition and sensitivity to oxidative damage. Biol Signals Recept 10: 93-111, 2001.

27. Arslan F, de Kleijn DP and Pasterkamp G: Innate immune signaling in cardiac ischemia. Nat Rev Cardiol 8: 292-300, 2011.

28. James AM, Hoogewijs K, Logan A, Hall AR, Ding S, Fearnley IM and Murphy MP: Non-enzymatic $\mathrm{N}$-acetylation of lysine residues by AcetylCoA often occurs via a proximal S-acetylated thiol intermediate sensitive to glyoxalase II. Cell Rep 18: 2105-2112, 2017.

29. Wagner GR, Bhatt DP, O'Connell TM, Thompson JW, Dubois LG, Backos DS, Yang H, Mitchell GA, Ilkayeva OR, Stevens RD, et al: A class of reactive Acyl-CoA species reveals the non-enzymatic origins of protein acylation. Cell Metab 25: 823-837.e8, 2017

30. Wagner GR and Hirschey MD: Nonenzymatic protein acylation as a carbon stress regulated by sirtuin deacylases. Mol Cell 54: $5-16,2014$.

31. Yang SJ, Choi JM, Kim L, Park SE, Rhee EJ, Lee WY, Oh KW, Park SW and Park CY: Nicotinamide improves glucose metabolism and affects the hepatic NAD-sirtuin pathway in a rodent model of obesity and type 2 diabetes. J Nutr Biochem 25: 66-72, 2014.

32. Carraro M and Bernardi P: Calcium and reactive oxygen species in regulation of the mitochondrial permeability transition and of programmed cell death in yeast. Cell Calcium 60: 102-107, 2016.

33. Giorgio V, Guo L, Bassot C, Petronilli V and Bernardi P: Calcium and regulation of the mitochondrial permeability transition. Cell Calcium 70: 56-63, 2018.

34. Nakagawa T, Shimizu S, Watanabe T, Yamaguchi O, Otsu K, Yamagata $\mathrm{H}$, Inohara $\mathrm{H}$, Kubo $\mathrm{T}$ and Tsujimoto $\mathrm{Y}$ : Cyclophilin D-dependent mitochondrial permeability transition regulates some necrotic but not apoptotic cell death. Nature 434: 652-658, 2005.

35. Martin JL, Gruszczyk AV, Beach TE, Murphy MP and Saeb-Parsy K: Mitochondrial mechanisms and therapeutics in ischaemia reperfusion injury. Pediatr Nephrol 34: 1167-1174, 2019.

36. Ong SB, Subrayan S, Lim SY, Yellon DM, Davidson SM and Hausenloy DJ: Inhibiting mitochondrial fission protects the heart against ischemia/reperfusion injury. Circulation 121: 2012-2022, 2010.

37. Din S, Mason M, Volkers M, Johnson B, Cottage CT, Wang Z, Joyo AY, Quijada P,Erhardt P, Magnuson NS, et al: Pim-1 preserves mitochondrial morphology by inhibiting dynamin-related protein 1 translocation. Proc Natl Acad Sci USA 110: 5969-5974, 2013.

38. Balaban RS: Cardiac energy metabolism homeostasis: Role of cytosolic calcium. J Mol Cell Cardiol 34: 1259-1271, 2002. 
39. Nan J, Zhu W, Rahman MS, Liu M, Li D, Su S, Zhang N, Hu X Yu H, Gupta MP and Wang J: Molecular regulation of mitochondrial dynamics in cardiac disease. Biochim Biophys Acta Mol Cell Res 1864: 1260-1273, 2017.

40. Sharp WW, Fang YH, Han M, Zhang HJ, Hong Z, Banathy A, Morrow E, Ryan JJ and Archer SL: Dynamin-related protein 1 (Drp1)-mediated diastolic dysfunction in myocardial ischemia-reperfusion injury: Therapeutic benefits of Drp1 inhibition to reduce mitochondrial fission. FASEB J 28: 316-326, 2014.

41. Murphy MP and Hartley RC: Mitochondria as a therapeutic target for common pathologies. Nat Rev Drug Discov 17: $865-886,2018$

42. Thomas LW and Ashcroft M: Exploring the molecular interface between hypoxia-inducible factor signalling and mitochondria. Cell Mol Life Sci 76: 1759-1777, 2019.

43. Song M, Mihara K, Chen Y, Scorrano L and Dorn GW II: Mitochondrial fission and fusion factors reciprocally orchestrate mitophagic culling in mouse hearts and cultured fibroblasts. Cell Metab 21: 273-286, 2015.

44. Chen Y, Liu Y and Dorn GW II: Mitochondrial fusion is essential for organelle function and cardiac homeostasis. Circ Res 109: 1327-1331, 2011

45. Song M, Gong G, Burelle Y, Gustafsson AB, Kitsis RN, Matkovich SJ and Dorn GW II: Interdependence of Parkin-Mediated mitophagy and mitochondrial fission in adult mouse hearts. Circ Res 117: 346-351, 2015.

46. Sabbah HN: Targeting the mitochondria in heart failure: A translational perspective. JACC Basic Transl Sci 5: 88-106, 2020.

47. Ikeda Y, Shirakabe A, Brady C, Zablocki D, Ohishi M and Sadoshima J: Molecular mechanisms mediating mitochondrial dynamics and mitophagy and their functional roles in the cardiovascular system. J Mol Cell Cardiol 78: 116-122, 2015.

48. Große L, Wurm CA, Bruser C, Neumann D, Jans DC and Jakobs S: Bax assembles into large ring-like structures remodeling the mitochondrial outer membrane in apoptosis. EMBO J 35: 402-413, 2016.

49. Kim H, Scimia MC, Wilkinson D, Trelles RD, Wood MR, Bowtell D, Dillin A, Mercola M and Ronai ZeA: Fine-tuning of Drp1/Fis1 availability by AKAP121/Siah2 regulates mitochondrial adaptation to hypoxia. Mol Cell 44: 532-544, 2011

50. Marin W: A-kinase anchoring protein 1 (AKAP1) and its role in some cardiovascular diseases. J Mol Cell Cardiol 138: 99-109, 2020

51. Disatnik MH, Ferreira JC, Campos JC, Gomes KS, Dourado PM Qi X and Mochly-Rosen D: Acute inhibition of excessive mitochondrial fission after myocardial infarction prevents long-term cardiac dysfunction. J Am Heart Assoc 2: e000461, 2013.

52. Ikeda Y, Shirakabe A, Maejima Y, Zhai P, Sciarretta S, Toli J, Nomura M, Mihara K, Egashira K, Ohishi M, et al: Endogenous Drp1 mediates mitochondrial autophagy and protects the heart against energy stress. Circ Res 116: 264-278, 2015.

53. Otera H, Wang C, Cleland MM, Setoguchi K, Yokota S, Youle RJ and Mihara K: Mff is an essential factor for mitochondrial recruitment of Drpl during mitochondrial fission in mammalian cells. J Cell Biol 191: 1141-1158, 2010.

54. Jin Q, Li R, Hu N, Xin T, Zhu P, Hu S, Ma S, Zhu H, Ren J and Zhou H: DUSP1 alleviates cardiac ischemia/reperfusion injury by suppressing the Mff-required mitochondrial fission and Bnip3-related mitophagy via the JNK pathways. Redox Biol 14 576-587, 2018

55. Li J, Li Y, Jiao J, Wang J, Li Y, Qin D and Li P: Mitofusin 1 is negatively regulated by microRNA 140 in cardiomyocyte apoptosis. Mol Cell Biol 34: 1788-1799, 2014.

56. Jiang X, Jiang H, Shen Z and Wang X: Activation of mitochondrial protease OMA1 by Bax and Bak promotes cytochrome c release during apoptosis. Proc Natl Acad Sci USA 111: 14782-14787, 2014.

57. Chistiakov DA, Shkurat TP, Melnichenko AA, Grechko AV and Orekhov AN: The role of mitochondrial dysfunction in cardiovascular disease: A brief review. Ann Med 50: 121-127, 2018

58. Minoia M, Boncoraglio A, Vinet J, Morelli FF, Brunsting JF Poletti A, Krom S, Reits E, Kampinga $\mathrm{HH}$ and Carra S: BAG3 induces the sequestration of proteasomal clients into cytoplasmic puncta: Implications for a proteasome-to-autophagy switch. Autophagy 10: 1603-1621, 2014.

59. Hammerling BC and Gustafsson AB: Mitochondrial quality control in the myocardium: Cooperation between protein degradation and mitophagy. J Mol Cell Cardiol 75: 122-130, 2014.

60. Kocaturk NM and Gozuacik D: Crosstalk between mammalian autophagy and the Ubiquitin-Proteasome system. Front Cell Dev Biol 6: 128, 2018.
61. Escobar-Henriques M, Altin S and Brave FD: Interplay between the ubiquitin proteasome system and mitochondria for protein homeostasis. Curr Issues Mol Biol 35: 35-58, 2020.

62. Nishida K and Otsu K: Sterile inflammation and degradation systems in heart failure. Circ J 81: 622-628, 2017.

63. Li J, Horak KM, Su H, Sanbe A, Robbins J and Wang X: Enhancement of proteasomal function protects against cardiac proteinopathy and ischemia/reperfusion injury in mice. J Clin Invest 121: 3689-3700, 2011.

64. Yu X and Kem DC: Proteasome inhibition during myocardial infarction. Cardiovasc Res 85: 312-320, 2010.

65. Zhou H and Toan S: Pathological roles of mitochondrial oxidative stress and mitochondrial dynamics in cardiac microvascular Ischemia/Reperfusion injury. Biomolecules 10: 85, 2020.

66. Morales PE, Arias-Duran C, Avalos-Guajardo Y, Aedo G, Verdejo HE, Parra V and Lavandero S: Emerging role of mitophagy in cardiovascular physiology and pathology. Mol Aspects Med 71: 100822, 2020.

67. Chen Y and Dorn GW II: PINK1-phosphorylated mitofusin 2 is a Parkin receptor for culling damaged mitochondria. Science 340: 471-475, 2013.

68. Siddall HK, Yellon DM, Ong SB, Mukherjee UA, Burke N, Hall AR, Angelova PR, Ludtmann MH, Deas E, Davidson SM, et al: Loss of PINK1 increases the heart's vulnerability to ischemia-reperfusion injury. PLoS One 8: e62400, 2013.

69. Kubli DA, Zhang X, Lee Y, Hanna RA, Quinsay MN, Nguyen CK, Jimenez R, Petrosyan S, Murphy AN and Gustafsson AB: Parkin protein deficiency exacerbates cardiac injury and reduces survival following myocardial infarction. J Biol Chem 288: 915-926, 2013.

70. Zhou H, Zhang Y, Hu S, Shi C, Zhu P, Ma Q, Jin Q, Cao F, Tian F and Chen Y: Melatonin protects cardiac microvasculature against ischemia/reperfusion injury via suppression of mitochondrial fission-VDAC1-HK2-mPTP-mitophagy axis. J Pineal Res 63: e12413, 2017.

71. Zhang T, Xue L, Li L, Tang C, Wan Z, Wang R, Tan J, Tan Y, Han $\mathrm{H}$, Tian R, et al: BNIP3 protein suppresses PINK1 kinase proteolytic cleavage to promote mitophagy. J Biol Chem 291: 21616-21629, 2016.

72. Wu W, Lin C, Wu K, Jiang L, Wang X, Li W, Zhuang H, Zhang X, Chen $\mathrm{H}$, Li S, et al: FUNDC1 regulates mitochondrial dynamics at the ER-mitochondrial contact site under hypoxic conditions. EMBO J 35: 1368-1384, 2016.

73. Tahrir FG, Knezevic T, Gupta MK, Gordon J, Cheung JY, Feldman AM and Khalili K: Evidence for the role of BAG3 in mitochondrial quality control in cardiomyocytes. J Cell Physiol 232: 797-805, 2017.

74. Schänzer A, Rupp S, Graf S, Zengeler D, Jux C, Akinturk H, Gulatz L, Mazhari N, Acker T, Van Coster R, et al: Dysregulated autophagy in restrictive cardiomyopathy due to Pro209Leu mutation in BAG3. Mol Genet Metab 123: 388-399, 2018.

75. Ong SB, Kalkhoran SB, Hernandez-Resendiz S, Samangouei P, Ong SG and Hausenloy DJ: Mitochondrial-shaping proteins in cardiac health and disease-the long and the short of it! Cardiovasc Drugs Ther 31: 87-107, 2017.

76. Kuznetsov AV and Margreiter R: Heterogeneity of mitochondria and mitochondrial function within cells as another level of mitochondrial complexity. Int J Mol Sci 10: 1911-1929, 2009.

77. Geng Y, Hu Y, Wang H, Shi S, Shi J and Qiu Z: Deficiency of interfibrillar mitochondria in post-acute myocardial infarction heart failure. Pak J Pharm Sci 30: 1089-1094, 2017.

78. Virani SS, Alonso A, Benjamin EJ, Bittencourt MS, Callaway CW, Carson AP, Chamberlain AM, Chang AR, Cheng S, Delling FN, et al: Heart disease and stroke statistics-2020 update: A report from the American heart association. Circulation 141: e139-e596, 2020.

79. Zhu SC, Chen C, Wu YN, Ahmed M, Kitmitto A, Greenstein AS, Kim SJ, Shao YF and Zhang YH: Cardiac complex II activity is enhanced by fat and mediates greater mitochondrial oxygen consumption following hypoxic re-oxygenation. Pflugers Arch 472: 367-374, 2020 .

80. Kohlhauer M, Pell VR, Burger N, Spiroski AM, Gruszczyk A, Mulvey JF, Mottahedin A, Costa ASH, Frezza C, Ghaleh B, et al: Protection against cardiac ischemia-reperfusion injury by hypothermia and by inhibition of succinate accumulation and oxidation is additive. Basic Res Cardiol 114: 18, 2019.

81. Turton JA, Fagg R, Sones WR, Williams TC and Andrews CM: Characterization of the myelotoxicity of chloramphenicol succinate in the B6C3F1 mouse. Int J Exp Pathol 87: 101-112, 2006. 
82. Ambekar CS, Lee JS, Cheung BM, Chan LC, Liang R and Kumana CR: Chloramphenicol succinate, a competitive substrate and inhibitor of succinate dehydrogenase: Possible reason for its toxicity. Toxicol In Vitro 18: 441-447, 2004.

83. Sala-Mercado JA, Wider J, Undyala VV, Jahania S, Yoo W, Mentzer RM Jr, Gottlieb RA and Przyklenk K: Profound cardioprotection with chloramphenicol succinate in the swine model of myocardial ischemia-reperfusion injury. Circulation 122 (11 Suppl): S179-S184, 2010.

84. Chouchani ET, Methner C, Nadtochiy SM, Logan A, Pell VR, Ding S, James AM, Cocheme HM, Reinhold J, Lilley KS, et al: Cardioprotection by S-nitrosation of a cysteine switch on mitochondrial complex I. Nat Med 19: 753-759, 2013.

85. Xu A, Szczepanek K, Hu Y, Lesnefsky EJ and Chen Q: Cardioprotection by modulation of mitochondrial respiration during ischemia-reperfusion: Role of apoptosis-inducing factor. Biochem Biophys Res Commun 435: 627-633, 2013.

86. Brand MD, Goncalves RL, Orr AL, Vargas L, Gerencser AA, Borch Jensen M, Wang YT, Melov S, Turk CN, Matzen JT, et al: Suppressors of superoxide- $\mathrm{H}_{2} \mathrm{O}_{2}$ production at site $\mathrm{I}(\mathrm{Q})$ of mitochondrial complex I protect against stem cell hyperplasia and ischemia-reperfusion injury. Cell Metab 24: 582-592, 2016.

87. Zozina VI, Covantev S, Goroshko OA, Krasnykh LM and Kukes VG: Coenzyme Q10 in cardiovascular and metabolic diseases: Current state of the problem. Curr Cardiol Rev 14: 164-174, 2018

88. Zhang ZW, Xu XC, Liu T and Yuan S: Mitochondrion-permeable antioxidants to treat ROS-Burst-mediated acute diseases. Oxid Med Cell Longev 2016: 6859523, 2016.

89. Di Lorenzo A, Iannuzzo G, Parlato A, Cuomo G, Testa C, Coppola M, D'Ambrosio G, Oliviero DA, Sarullo S, Vitale G, et al: Clinical evidence for Q10 coenzyme supplementation in heart failure: From energetics to functional improvement. J Clin Med 9: 1266, 2020.

90. Mortensen AL, Rosenfeldt F and Filipiak KJ: Effect of coenzyme Q10 in Europeans with chronic heart failure: A sub-group analysis of the Q-SYMBIO randomized double-blind trial. Cardiol J 26: 147-156, 2019.

91. Reily C, Mitchell T, Chacko BK, Benavides G, Murphy MP and Darley-Usmar V: Mitochondrially targeted compounds and their impact on cellular bioenergetics. Redox Biol 1: 86-93, 2013.

92. Smith RA and Murphy MP: Animal and human studies with the mitochondria-targeted antioxidant MitoQ. Ann N Y Acad Sci 1201: 96-103, 2010.

93. Lee FY, Shao PL, Wallace CG, Chua S, Sung PH, Ko SF, Chai HT, Chung SY, Chen KH, Lu HI, et al: Combined therapy with SS31 and mitochondria mitigates myocardial ischemia-reperfusion injury in rats. Int J Mol Sci 19: 2782, 2018.

94. Kloner RA, Hale SL, Dai W, Gorman RC, Shuto T, Koomalsingh KJ, Gorman JH III, Sloan RC, Frasier CR, Watson CA, et al: Reduction of ischemia/reperfusion injury with bendavia, a mitochondria-targeting cytoprotective peptide. J Am Heart Assoc 1: e001644, 2012.

95. Botker HE, Cabrera-Fuentes HA, Ruiz-Meana M, Heusch G and Ovize M: Translational issues for mitoprotective agents as adjunct to reperfusion therapy in patients with ST-segment elevation myocardial infarction. J Cell Mol Med 24: 2717-2729, 2020

96. Mailloux RJ: Application of mitochondria-targeted pharmaceuticals for the treatment of heart disease. Curr Pharm Des 22: 4763-4779, 2016

97. van Empel VP, Bertrand AT, van Oort RJ, van der Nagel R, Engelen M, van Rijen HV, Doevendans PA, Crijns HJ, Ackerman SL, Sluiter W and De Windt LJ: EUK-8, a superoxide dismutase and catalase mimetic, reduces cardiac oxidative stress and ameliorates pressure overload-induced heart failure in the harlequin mouse mutant. J Am Coll Cardiol 48: 824-832, 2006.

98. Baguisi A, Casale RA, Kates SA, Lader AS, Stewart K and Beeuwkes R III: CMX-2043 efficacy in a rat model of cardiac ischemia-reperfusion injury. J Cardiovasc Pharmacol Ther 21: 563-569, 2016

99. Tsubota K: The first human clinical study for NMN has started in Japan. NPJ Aging Mech Dis 2: 16021, 2016.

100. Hong W, Mo F, Zhang Z, Huang M and Wei X: Nicotinamide mononucleotide: A promising molecule for therapy of diverse diseases by targeting NAD+ metabolism. Front Cell Dev Biol 8 : 246,2020

101. Irie J, Inagaki E, Fujita M, Nakaya H, Mitsuishi M, Yamaguchi S, Yamashita K, Shigaki S, Ono T, Yukioka H, et al: Effect of ora administration of nicotinamide mononucleotide on clinical parameters and nicotinamide metabolite levels in healthy Japanese men. Endocr J 67: 153-160, 2020.
102. Bendickova $\mathrm{K}$, Tidu $\mathrm{F}$ and Fric J: Calcineurin-NFAT signalling in myeloid leucocytes: New prospects and pitfalls in immunosuppressive therapy. EMBO Mol Med 9: 990-999, 2017.

103. Ottani F, Latini R, Staszewsky L, La Vecchia L, Locuratolo N, Sicuro M, Masson S, Barlera S, Milani V, Lombardi M, et al: Cyclosporine a in reperfused myocardial infarction: The multicenter, controlled, open-label CYCLE trial. J Am Coll Cardiol 67: 365-374, 2016.

104. Ikeda G, Matoba T, Nakano Y, Nagaoka K, Ishikita A, Nakano K, Funamoto D, Sunagawa K and Egashira K: Nanoparticle-mediated targeting of cyclosporine a enhances cardioprotection against ischemia-reperfusion injury through inhibition of mitochondrial permeability transition pore opening. Sci Rep 6: 20467, 2016.

105. Jahandiez V, Cour M, Bochaton T, Abrial M, Loufouat J, Gharib A, Varennes A, Ovize M and Argaud L: Fast therapeutic hypothermia prevents post-cardiac arrest syndrome through cyclophilin D-mediated mitochondrial permeability transition inhibition. Basic Res Cardiol 112: 35, 2017.

106. Parodi-Rullán RM, Soto-Prado J, Vega-Lugo J, Chapa-Dubocq X, Díaz-Cordero SI and Javadov S: Divergent effects of cyclophilin-D inhibition on the female rat heart: Acute versus chronic post-myocardial infarction. Cell Physiol Biochem 50: 288-303, 2018.

107. Khuchua Z, Glukhov AI, Strauss AW and Javadov S: Elucidating the beneficial role of PPAR agonists in cardiac diseases. Int J Mol Sci 19: 3464, 2018

108. Lalloyer F and Staels B: Fibrates, glitazones, and peroxisome proliferator-activated receptors. Arterioscler Thromb Vasc Biol 30: 894-899, 2010

109. Viscomi C, Bottani E, Civiletto G, Cerutti R, Moggio M, Fagiolari $\mathrm{G}$, Schon EA, Lamperti $\mathrm{C}$ and Zeviani M: In vivo correction of COX deficiency by activation of the AMPK/PGC-1 $\alpha$ axis. Cell Metab 14: 80-90, 2011.

110. Madrid-Miller A, Moreno-Ruiz LA, Borrayo-Sánchez G, Almeida-Gutiérrez E, Martínez-Gómez DF and Jáuregui-Aguilar R: Ipact of bezafibrate treatment in patients with hyperfibrinogenemia and ST-elevation acute myocardial infarction: A randomized clinical trial. Cir Cir 78: 229-237, 2010.

111. Kernan WN, Inzucchi SE, Viscoli CM, Brass LM, Bravata DM, Shulman GI, McVeety JC and Horwitz RI: Pioglitazone improves insulin sensitivity among nondiabetic patients with a recent transient ischemic attack or ischemic stroke. Stroke 34: 1431-1436, 2003.

112. Liu J and Wang LN: Peroxisome proliferator-activated receptor gamma agonists for preventing recurrent stroke and other vascular events in people with stroke or transient ischaemic attack. Cochrane Database Syst Rev 12: CD010693, 2017.

113. Palee S, Weerateerangkul P, Surinkeaw S, Chattipakorn S and Chattipakorn N: Effect of rosiglitazone on cardiac electrophysiology, infarct size and mitochondrial function in ischaemia and reperfusion of swine and rat heart. Exp Physiol 96: 778-789, 2011.

114. Nissen SE and Wolski K: Effect of rosiglitazone on the risk of myocardial infarction and death from cardiovascular causes. $\mathrm{N}$ Engl J Med 356: 2457-2471, 2007.

115. Sunaga H, Koitabashi N, Iso T, Matsui H, Obokata M, Kawakami R, Murakami M, Yokoyama T and Kurabayashi M: Activation of cardiac AMPK-FGF21 feed-forward loop in acute myocardial infarction: Role of adrenergic overdrive and lipolysis byproducts. Sci Rep 9: 11841, 2019.

116. Zhang DS, Liang GY, Liu DX, Yu J and Wang F: Role of phosphorylated AMP-activated protein kinase (AMPK) in myocardial insulin resistance after myocardial ischemia-reperfusion during cardiopulmonary bypass surgery in dogs. Med Sci Monit 25: 4149-4158, 2019

117. Zhou J, Massey S, Story D and Li L: Metformin: An old drug with new applications. Int J Mol Sci 19: 2863, 2018.

118. Chin JT, Troke JJ, Kimura N, Itoh S, Wang X, Palmer OP, Robbins RC and Fischbein MP: A novel cardioprotective agent in cardiac transplantation: Metformin activation of AMP-activated protein kinase decreases acute ischemia-reperfusion injury and chronic rejection. Yale J Biol Med 84: 423-432, 2011.

119. Palee S, Higgins L, Leech T, Chattipakorn SC and Chattipakorn N: Acute metformin treatment provides cardioprotection via improved mitochondrial function in cardiac ischemia / reperfusion injury. Biomed Pharmacother 130: 110604, 2020.

120. Holman RR, Paul SK, Bethel MA, Matthews DR and Neil HA: 10 -Year follow-up of intensive glucose control in type 2 diabetes. N Engl J Med 359: 1577-1589, 2008. 
121. Mellbin LG, Malmberg K, Norhammar A, Wedel H and Rydén L; DIGAMI 2 Investigators: The impact of glucose lowering treatment on long-term prognosis in patients with type 2 diabetes and myocardial infarction: A report from the DIGAMI 2 trial. Eur Heart J 29: 166-176, 2008.

122. Hartman MHT, Prins JKB, Schurer RAJ, Lipsic E, Lexis CPH, van der Horst-Schrivers ANA, van Veldhuisen DJ, van der Horst ICC and van der Harst P: Two-year follow-up of 4 months metformin treatment vs. placebo in ST-elevation myocardia infarction: Data from the GIPS-III RCT. Clin Res Cardiol 106 939-946, 2017.

123. Whitaker RM, Corum D, Beeson CC and Schnellmann RG: Mitochondrial biogenesis as a pharmacological target: A new approach to acute and chronic diseases. Annu Rev Pharmacol Toxicol 56: 229-249, 2016

124. Kim H, Lee JY, Park KJ, Kim WH and Roh GS: A mitochondrial division inhibitor, Mdivi-1, inhibits mitochondrial fragmentation and attenuates kainic acid-induced hippocampal cell death. BMC Neurosci 17: 33, 2016.

125. Veeranki S and Tyagi SC: Mdivi-1 induced acute changes in the angiogenic profile after ischemia-reperfusion injury in female mice. Physiol Rep 5: e13298, 2017.

126. Gao G, Chen W, Yan M, Liu J, Luo H, Wang C and Yang P. Rapamycin regulates the balance between cardiomyocyte apoptosis and autophagy in chronic heart failure by inhibiting mTOR signaling. Int J Mol Med 45: 195-209, 2020.

127. Wang JX, Jiao JQ, Li Q, Long B, Wang K, Liu JP, Li YR and Li PF: miR-499 regulates mitochondrial dynamics by targeting calcineurin and dynamin-related protein-1. Nat Med 17: 71-78, 2011.

128. Babot M, Birch A, Labarbuta P and Galkin A: Characterisation of the active/de-active transition of mitochondrial complex I. Biochim Biophys Acta 1837: 1083-1092, 2014.

129. Gorenkova N, Robinson E, Grieve DJ and Galkin A: Conformational change of mitochondrial complex I increases ROS sensitivity during ischemia. Antioxid Redox Signal 19: 1459-1468, 2013.

130. Chouchani ET, James AM, Methner C, Pell VR, Prime TA, Erickson BK, Forkink M, Lau GY, Bright TP, Menger KE, et al Identification and quantification of protein S-nitrosation by nitrite in the mouse heart during ischemia. J Biol Chem 292: 14486-14495, 2017

131. Pan J and Carroll KS: Light-mediated sulfenic acid generation from photocaged cysteine sulfoxide. Org Lett 17: 6014-6017, 2015.

132. Ukuwela AA, Bush AI, Wedd AG and Xiao Z: Reduction potentials of protein disulfides and catalysis of glutathionylation and deglutathionylation by glutaredoxin enzymes. Biochem J 474: 3799-3815, 2017.

133. Kolluru GK, Shen X and Kevil CG: Reactive sulfur species: A new redox player in cardiovascular pathophysiology. Arterioscler Thromb Vasc Biol 40: 874-884, 2020.

134. Davalli P, Mitic T, Caporali A, Lauriola A and D'Arca D: ROS, cell senescence, and novel molecular mechanisms in aging and age-related diseases. Oxid Med Cell Longev 2016: 3565127, 2016.

135. Shahzad S, Hasan A, Faizy AF, Mateen S, Fatima N and Moin S: Elevated DNA damage, oxidative stress, and impaired response defense system inflicted in patients with myocardial infarction. Clin Appl Thromb Hemost 24: 780-789, 2018.

136. Dey S, DeMazumder D, Sidor A, Foster DB and O'Rourke B: Mitochondrial ROS drive sudden cardiac death and chronic proteome remodeling in heart failure. Circ Res 123: 356-371, 2018.
137. Gottlieb RA and Thomas A: Mitophagy and mitochondrial quality control mechanisms in the heart. Curr Pathobiol Rep 5: 161-169, 2017.

138. Guan R, Zou W, Dai X, Yu X, Liu H, Chen Q and Teng W: Mitophagy, a potential therapeutic target for stroke. J Biomed Sci 25: 87, 2018

139. Andres AM, Tucker KC, Thomas A, Taylor DJ, Sengstock D, Jahania SM, Dabir R, Pourpirali S, Brown JA, Westbrook DG, et al: Mitophagy and mitochondrial biogenesis in atrial tissue of patients undergoing heart surgery with cardiopulmonary bypass. JCI Insight 2: e89303, 2017.

140. Moyzis AG, Sadoshima J and Gustafsson AB: Mending a broken heart: The role of mitophagy in cardioprotection. Am J Physiol Heart Circ Physiol 308: H183-H192, 2015.

141. Ding S, Wu D, Lu Q, Qian L, Ding Y, Liu G, Jia X, Zhang Y, Xiao W and Gong W: Angiopoietin-like 4 deficiency upregulates macrophage function through the dysregulation of cell-intrinsic fatty acid metabolism. Am J Cancer Res 10: 595-609, 2020.

142. Cai J, Wang D, Zhao FQ, Liang S and Liu J: AMPK-mTOR pathway is involved in glucose-modulated amino acid sensing and utilization in the mammary glands of lactating goats J Anim Sci Biotechnol 11: 32, 2020.

143. Mackenzie RM, Salt IP, Miller WH, Logan A, Ibrahim HA, Degasperi A, Dymott JA, Hamilton CA, Murphy MP, Delles C and Dominiczak AF: Mitochondrial reactive oxygen species enhance AMP-activated protein kinase activation in the endothelium of patients with coronary artery disease and diabetes. Clin Sci (Lond) 124: 403-411, 2013.

144. Zhao Y, Shang F, Shi W, Zhang J, Liu X, Li B, Hu X and Wang L: Angiotensin II receptor type 1 antagonists modulate vascular smooth muscle cell proliferation and migration via AMPK/mTOR. Cardiology 143: 1-10, 2019.

145. Gerczuk PZ and Kloner RA: An update on cardioprotection: A review of the latest adjunctive therapies to limit myocardial infarction size in clinical trials. J Am Coll Cardiol 59: 969-978, 2012.

146. Obtułowicz K: Bradykinin-mediated angioedema. Pol Arch Med Wewn 126: 76-85, 2016.

147. Taddei S and Bortolotto L: Unraveling the pivotal role of bradykinin in ACE inhibitor activity. Am J Cardiovasc Drugs 16 309-321, 2016.

148. Lepelley M, Bernardeau C, Defendi F, Crochet J, Mallaret M and Bouillet L: Update on bradykinin-mediated angioedema in 2020. Therapie 75: 195-205, 2020.

149. Koh JQS, Fernando H, Peter K and Stub D: Opioids and ST elevation myocardial infarction: A systematic review. Heart Lung Circ 28: 697-706, 2019.

150. Tavenier AH, Hermanides RS, Ottervanger JP, Ter Horst PGJ, Kedhi E and van 't Hof AWJ: Risks of opioids in ST-elevation myocardial infarction: A review. Drug Saf 41: 1303-1308, 2018

151. Jones SP, Tang XL, Guo Y, Steenbergen C, Lefer DJ, Kukreja RC, Kong M, Li Q, Bhushan S, Zhu X, et al: The NHLBI-sponsored consortium for preclinicAl assESsment of cARdioprotective therapies (CAESAR): A new paradigm for rigorous, accurate, and reproducible evaluation of putative infarct-sparing interventions in mice, rabbits, and pigs. Circ Res 116: 572-586, 2015.

(i) $(-)$ This work is licensed under a Creative Commons Attribution-NonCommercial-NoDerivatives 4.0 International (CC BY-NC-ND 4.0) License. 